\title{
3D Wave-equation Dispersion Inversion of Surface Waves Recorded on Irregular Topography
}

\author{
Zhaolun Liu*, Jing Li ${ }^{\dagger}$, Sherif M. Hanafy ${ }^{\ddagger}$, Kai Lu* and Gerard Schuster* \\ * Department of Earth Science and Engineering, \\ King Abdullah University of Science and Technology, \\ Thuwal, Saudi Arabia, 23955-6900. \\ Email: zhaolun.liu@kaust.edu.sa; gerard.schuster@kaust.edu.sa. \\ $\dagger$ Formerly King Abdullah University of Science and Technology, Department of Earth \\ Science and Engineering, Thuwal, Saudi Arabia; presently College of Geo-Exploration \\ Science and Technology, Jilin University, Changchun 130021, China. E-mail: \\ inter.lijing@gmail.com \\ $\ddagger$ Formerly King Abdullah University of Science and Technology, Department of Earth \\ Science and Engineering, Thuwal, Saudi Arabia; presently King Fahd University of \\ Petroleum and Minerals, College of Petroleum Engineering and Geosciences, Dhahran, \\ Saudi Arabia. E-mail:sherif.geo@gmail.com
}

(December 6, 2019)

Running head: 3D TWD

\begin{abstract}
Irregular topography can cause strong scattering and defocusing of propagating surface waves, so it is important to account for such effects when inverting surface waves for the shallow S-velocity structures. We now present a 3D surface-wave dispersion inversion method that takes into account the topographic effects modeled by a 3D spectral element solver.
\end{abstract}


The objective function is the frequency summation of the squared wavenumber differences $\Delta \kappa(\omega)^{2}$ along each azimuthal angle of the fundamental mode or higher-order modes of Rayleigh waves in each shot gather. The wavenumbers $\kappa(\omega)$ associated with the dispersion curves are calculated using the data recorded along the irregular free surface. Numerical tests on both synthetic and field data demonstrate that 3D topographic wave equation dispersion inversion (TWD) can accurately invert for the S-velocity model from surface-wave data recorded on irregular topography. Field data tests for data recorded across an Arizona fault suggest that, for this example, the 2D TWD can be as accurate as the 3D tomographic model. This suggests that in some cases the 2D TWD inversion is preferred over 3D TWD because of its significant reduction in computational costs. Compared to the 3-D P-wave velocity tomogram, the 3D S-wave tomogram agrees much more closely with the geological model taken from the trench log. The agreement with the trench log is even better when the $\mathrm{Vp} /$ Vs tomogram is computed, which reveals a sharp change in velocity across the fault. The localized velocity anomaly in the $\mathrm{Vp} / \mathrm{Vs}$ tomogram is in very good agreement with the well log. Our results suggest that integrating the Vp and Vs tomograms can sometimes give the most accurate estimates of the subsurface geology across normal faults. 


\section{INTRODUCTION}

Irregular topography is known to have a significant impact on the amplitudes and phases of propagating surface waves (Snieder, 1986; Fu and Wu, 2001). Ignoring topography in surface wave inversion can lead to significant errors in the inverted model. Moreover, it is expected that the 2D assumptions about the subsurface model cannot fully approximate wave propagation in the presence of significant 3D variations in topography. In these cases, it is important to employ a 3D surface-wave inversion method that fully accounts for wave propagation along irregular topography.

Eguiluz and Maradudin (1983) and Mayer et al. (1991) analytically studied the effect of surface roughness on the dispersion relations of a Rayleigh wave propagating in an isotropic medium with randomly rough surfaces. For significant topographic variations on a wavelength scale, they showed that the relief of the free surface induces attenuation of amplitudes, reduces the phase velocity (Eguiluz and Maradudin, 1983) and generates both Love waves and higher-order modes of Rayleigh waves (Mayer et al., 1991). These authors argued that these waves sense the uppermost part of the model as an upper layer with a reduced effective velocity.

When the wavelength is much smaller than the characteristic length scale of the topographic relief, the source-receiver distance factor may play a significant role. The is especially true for the fundamental mode of the Rayleigh waves whose propagation is strongly influenced by the free surface (Köhler et al., 2012). Köhler et al. (2012) empirically investigated the effect of topography on the propagation of short-period Rayleigh waves by elastic simulations with a spectral element code and a 3-D model with significant topographical variations. They showed that topography along a profile could result in an underestimation 
of the phase velocities associated with the surface waves.

Accounting for topography is also essential for full waveform inversion (FWI) of surface waves (Borisov et al., 2018). Nuber et al. (2016) and Pan et al. (2018) use simulations to demonstrate that even minor topographic variations of the free surface will have a significant effect in the accuracy of FWI. They found that neglecting topography with an elevation fluctuation greater than half the minimum seismic wavelength leads to significant errors in the inverted image (Nuber et al., 2016).

Li and Schuster (2016) developed a wave equation dispersion inversion (WD) method for inverting dispersion curves associated with surface waves. Li et al. (2019a) applied WD to Love waves and Liu et al. (2019) extended it to the 3D case, which includes the multi-scale and layer-stripping WD proposed by Liu and Huang (2019). Empirical evidence suggests that WD has the benefit of robust convergence compared to the tendency of FWI (Groos et al., 2014; Pérez Solano et al., 2014; Dou and Ajo-Franklin, 2014; Yuan et al., 2015; Groos et al., 2017) to getting stuck in a local minimum. It has the advantage over the traditional inversion of dispersion curves (Haskell, 1953; Xia et al., 1999, 2002; Park et al., 1999) in that it does not assume a layered model and is valid for arbitrary 2D or 3D media. Later, Li et al. (2017b, 2019b) developed 2D topographic WD (i.e., topographic WD, also denoted as TWD) which incorporates the free-surface topography into the finite-difference solutions of the elastic wave equation. Our new paper now extends $2 \mathrm{D}$ TWD to the $3 \mathrm{D}$ case. To account for strong variations in topography, we use the elastic modeling code SPECFEM3D based on the spectral-element method (SEM) (Komatitsch and Vilotte, 1998; Komatitsch and Tromp, 1999). The inversion algorithm is written in the format of SeisFlows, an open source Python package that can interface with SPECFEM3D (Modrak et al., 2018). 
After the introduction, we describe the theory of 3D TWD and its implementation. We also discuss how to calculate the source-receiver offset distance along a 3D irregular surface, which is used to calculate the dispersion curves of the data recorded on the irregular surface. Numerical tests on synthetic data are presented in the third section to validate the theory. The field data test is for 3D vertical-component data recorded over a normal fault located near the Arizona-Utah border. Finally, the discussion and conclusions are given in the fourth and last sections.

\section{THEORY}

We first present the mathematical theory for 3D TWD, following the derivation of Liu et al. (2019), except it is for a 3D irregular surface. Then, we show how to calculate the source-receiver distance on a 3D irregular surface. Finally, the workflow of 3D TWD is given.

\section{Theory of 3D TWD}

The basic theory of 3D TWD is the same for 3D WD (Liu et al., 2018, 2019), except a 3D topographic surface is now included in the formulation. The wave-equation dispersion inversion method inverts for the S-wave velocity model to minimize the dispersion objective function

$$
\varepsilon=\frac{1}{2} \sum_{\omega} \sum_{\theta}[\overbrace{\kappa(\theta, \omega)_{\text {pre }}-\kappa(\theta, \omega)_{o b s}}^{\text {residual }=\Delta \kappa(\theta, \omega)}]^{2},
$$

where $\kappa(\omega, \theta)_{\text {pre }}$ represents the predicted dispersion curve picked from the simulated spectrum along the azimuth angle $\theta$, and $\kappa(\omega, \theta)_{\text {obs }}$ describes the observed dispersion curve obtained from the recorded spectrum along the azimuth $\theta$. In the $2 \mathrm{D}$ case, the azimuthal 
angles have only two values: $0^{\circ}$ and $180^{\circ}$, corresponding to the left and right directions, respectively.

The gradient $\gamma(\boldsymbol{x})$ of $\varepsilon$ with respect to the S-wave velocity $v_{s}(\boldsymbol{x})$ is given by Liu et al. (2018, 2019):

$$
\begin{aligned}
& \gamma(\boldsymbol{x})=\frac{\partial \varepsilon}{\partial v_{s}(\boldsymbol{x})}=-\sum_{\omega} 4 v_{s 0}(\boldsymbol{x}) \rho_{0}(\boldsymbol{x}) \mathfrak{R}\{ \\
& \overbrace{\int \sum_{\theta} \frac{1}{A(\theta, \omega)} \Delta \kappa(\theta, \omega) \hat{D}(\boldsymbol{g}, \theta, \omega)_{o b s}^{*} G_{3 k, k}(\boldsymbol{g} \mid \boldsymbol{x}) d \boldsymbol{g}}^{\text {backprojected data }=B_{k, k}(\boldsymbol{x}, \omega)^{*}} \overbrace{D_{j, j}(\boldsymbol{x}, \omega)}^{\text {source }=f_{j, j}(\boldsymbol{x}, \omega)} \\
& \overbrace{-\frac{1}{2} \int \sum_{\theta} \frac{1}{A(\theta, \omega)} \Delta \kappa(\theta, \omega) \hat{D}(\boldsymbol{g}, \theta, \omega)_{o b s}^{*} G_{3 n, k}(\boldsymbol{g} \mid \boldsymbol{x}) d \boldsymbol{g}}^{\text {backprojected data } \overbrace{\left[D_{n, k}(\boldsymbol{x}, \omega)^{*}\right.}(\boldsymbol{x}, \omega)+D_{n, k}(\boldsymbol{x}, \omega)]}\}
\end{aligned}
$$

where $v_{s 0}(\boldsymbol{x})$ and $\rho_{0}(\boldsymbol{x})$ are the reference S-velocity and density distributions at location $\boldsymbol{x}$, respectively, and $A(\theta, \omega)$ is given in Liu et al. (2019). $D_{i}(\boldsymbol{x}, \omega)$ denotes the $i^{\text {th }}$ component of the particle velocity recorded at $\boldsymbol{x}$ resulting from a vertical-component force. Einstein notation is assumed in equation 2 , where $D_{i, j}=\frac{\partial D_{i}}{\partial_{x_{j}}}$ for $i, j \in\{1,2,3\}$. The $3 \mathrm{D}$ harmonic Green's tensor $G_{3 j}(\boldsymbol{g} \mid \boldsymbol{x})$ is the particle velocity at location $\boldsymbol{g}$ along the $j^{\text {th }}$ direction resulting from a vertical-component source at $\boldsymbol{x}$ in the reference medium. The term $f_{i, j}(\boldsymbol{x}, \omega)$ for $i$ and $j \in\{1,2,3\}$ represents the downgoing source field at $\boldsymbol{x}$, and $B_{i, j}(\boldsymbol{x}, \boldsymbol{s}, \omega)$ for $i$ and $j \in\{1,2,3\}$ denotes the backprojected scattered field at $\boldsymbol{x} . \hat{D}(\boldsymbol{g}, \theta, \omega)_{\text {obs }}^{*}$ represents the weighted conjugated data defined as

$$
\hat{D}(\boldsymbol{g}, \theta, \omega)_{o b s}^{*}=2 \pi i \boldsymbol{g} \cdot \boldsymbol{n} e^{i \boldsymbol{g} \cdot \boldsymbol{n} \Delta \kappa} \int_{C} D\left(\boldsymbol{g}^{\prime}, \omega\right)_{o b s}^{*} d \boldsymbol{g}^{\prime}
$$

where $\boldsymbol{n}=(\cos \theta, \sin \theta)$ and $C$ is the line $\left(\boldsymbol{g}^{\prime}-\boldsymbol{g}\right) \cdot \boldsymbol{n}=0$. The above equation indicates that the gradient is computed using a weighted zero-lag correlation between the source and backward-extrapolated receiver wavefields.

The optimal S-wave velocity model $v_{s}(\boldsymbol{x})$ is obtained using the steepest-descent formula 
(Nocedal and Wright, 2006)

$$
v_{s}(\boldsymbol{x})^{(k+1)}=v_{s}(\boldsymbol{x})^{(k)}-\alpha \gamma(\boldsymbol{x}),
$$

where $\alpha$ is the step length and the superscript $(k)$ denotes the $k^{t h}$ iteration. In practice a preconditoned conjugate gradient method can be used for faster convergence (Nocedal and Wright, 2006).

\section{Source-receiver Distance on a 3D Irregular Surface}

When the wavelength is smaller than the characteristic wavelength of the topographical relief, the source-receiver distance factor will play a significant role in the accuracy of the final tomogram (Köhler et al., 2012). Thus, we should calculate the source-receiver offset distance along the actual irregular surface instead of assuming it to be a flat surface.

For the flat free surface shown in Figure 1a, the source-receiver offset $l$ along the surface is the length of the line segment $\mathbf{s r}_{\mathbf{1}}$, which is the same as the Euclidean distance $l_{e}$ between the source at $\mathbf{s}$ and the receiver at $\mathbf{r}_{1}$. When the surface is irregular as shown in Figure $1 \mathrm{~b}$, the source-receiver offset $l$ along the surface is the length of the segment of a curve on the surface, which is larger than the Euclidean distance $l_{e}$. The source-receiver offset distance along the irregular surface is called the "geodesic distance", which is the shortest route between two points on the surface. Appendix A introduces the method for calculating the geodesic distance on a triangular mesh surface.

Figure 2 shows the offset $L$ and azimuth $\theta$ associated with the source at $\mathbf{s}$ to the receiver at $\mathbf{r}$ on an irregular surface. Here, the azimuth is along the direction from $\mathbf{s}^{\prime}$ to $\mathbf{r}^{\prime}$, where $\mathbf{s}^{\prime}$ and $\mathbf{r}^{\prime}$ are the perpendicular projections of points $\mathbf{s}$ and $\mathbf{r}$ on the plane $z=0$, respectively. Once we get the offset and azimuth for the receivers, we can calculate the dispersion curve of the shot gather by applying to the common shot gather (CSG) the discrete Radon transform 
in the frequency domain as presented in Appendix B.

[Figure 1 about here.]

[Figure 2 about here.]

\section{Workflow of 3D TWD}

The workflow for implementing 3D TWD is summarized by the following steps.

1. Remove the first-arrival body waves and higher-order modes of the Rayleigh waves in the shot gathers (Li et al., 2017a).

2. Determine the source-receiver offset along the irregular surface, and the range of the dominant azimuth angles $\theta$ for each shot gather. The dominant azimuth angle is defined in Liu et al. (2019).

3. Apply a discrete Radon transform followed by the temporal Fourier transform of the predicted and observed common shot gathers to compute the dispersion curves $\kappa(\theta, \omega)$ and $\kappa(\theta, \omega)_{o b s}$ along each azimuthal angle $\theta$. Calculate the sum of the squared dispersion residuals in equation 1.

4. Calculate the weighted data $\hat{D}(\boldsymbol{g}, \omega)_{o b s}^{*}$ according to equation 3 . The source-side and receiver-side wavefields in equation 2 are computed by the SEM solution to the 3D elastic wave equation.

5. Calculate and sum the gradients for all the shot gathers. The source illumination is sometimes needed as a preconditioner (Plessix and Mulder, 2004). 
6. Calculate the step length and update the S-wave tomogram using the steepest-descent or conjugate gradient methods. In practice, we use a preconditioned conjugate gradient method.

\section{NUMERICAL RESULTS}

The effect of topography on the calculation of the dispersion curves is first tested for data computed over a homogeneous half-space model with an irregular free surface. Then the effectiveness of 3D TWD is evaluated with synthetic and field data examples. The data are associated with 1) a simple checkerboard model, 2) the complex 3D Foothills model and a surface seismic experiment carried out in the Washington fault zone of northern Arizona, U.S.

In the synthetic examples, the observed and predicted data are generated by a spectralelement solver SPECFEM3D (Komatitsch and Vilotte, 1998; Komatitsch and Tromp, 1999). The mesh is generated by the software package CUBIT, which is a software toolkit for robust generation of two- and three-dimensional finite element meshes (grids) and geometry preparation. For 3D TWD, only the S-wave velocity model is inverted and the true $\mathrm{P}$-wave velocity model is used for modeling the predicted surface waves. The density model is

homogeneous with $\rho=2000 \mathrm{~kg} / \mathrm{m}^{3}$ for all synthetic data tests. The source wavelet is a Ricker wavelet.

\section{Homogeneous Half Space}

The topography shown in Figure 2 is used for testing the effect of topography on the calculation of the dispersion curves associated with Rayleigh waves. The study area is 150 
$\mathrm{m}$ in the $\mathrm{x}$-direction and $220 \mathrm{~m}$ in the $\mathrm{y}$-direction. The maximum elevation difference of the topography is $36 \mathrm{~m}$. We choose a homogeneous medium $\left(v_{s}=1 \mathrm{~km} / \mathrm{s}, v_{p}=\sqrt{3} v_{s}, \rho=2300\right.$ $\mathrm{kg} / \mathrm{m}^{3}$ ) with a free surface on the top. There are 1024 receivers represented by the red dots in Figure 3a, which are arranged in 32 parallel lines where each line has 32 receivers. A vertical-component shot is fired at the location A in Figure 3a. The peak frequency of the source wavelet is $30 \mathrm{~Hz}$.

The data recorded by the receivers within the yellow area in Figure 3a are chosen for analysis, and the geodesic paths from the source at A are shown Figure 3b. Figure 3c shows the differences between the geodesic and Euclidean distances for these receivers, which indicates that the source-receiver distance errors introduced by assuming a flat surface are up to $12 \mathrm{~m}$ for the far-offset receivers. Such source-receiver distance errors will lead to inaccurate estimates of the phase velocity of surface waves, which can be seen in the following tests. The seismograms recorded from these receivers are displayed as the red wiggles in Figure 3d, where the seismograms from the flat-surface model (blue) are displayed for comparison.

We apply the discrete Radon transform in the frequency domain to the seismograms in Figure 3d to get their dispersion images shown in Figure 4. We then pick the dispersion curves shown as the red curves in Figure 4. Here, the Euclidean and geodesic distances are used in Figures 4a and 4b, respectively, and the theoretical dispersion curves are represented by the green curves. The dispersion image computed from the data recorded in the flatsurface model is shown in Figure 4c for comparison. We can see that the dispersion curves calculated by using the geodesic distances are more accurate than those calculated by the Euclidean distances. 
Figure 5 shows the dispersion curves for the azimuths ranged from $0^{\circ}$ to $360^{\circ}$ computed from the CSGs recorded in the flat-surface model, where the black dashed lines represent their contours which are the reference contours. The cyan dash-dot and red lines in Figure 5 represent the contours of the dispersion curves from the topographic model calculated by the Euclidean and geodesic distances, respectively. The contour calculated by the geodesic distance is much closer to the reference contour compared to the ones computed by the Euclidean distance, especially for the frequencies between 35 and $60 \mathrm{~Hz}$.

[Figure 3 about here.]

[Figure 4 about here.]

[Figure 5 about here.]

\section{Checkerboard Test}

The 3D checkerboard model is shown in Figure 6a, and its vertical slices at $y=80 \mathrm{~m}$ and $160 \mathrm{~m}$ are shown in Figures 7a and 7c, respectively. We use the same topography and acquisition geometry as those used in the homogeneous half-space test. The values of the high and low S-velocities are $1100 \mathrm{~m} / \mathrm{s}$ and $900 \mathrm{~m} / \mathrm{s}$, respectively. The initial S-velocity model is homogeneous with $v_{s}=1000 \mathrm{~m} / \mathrm{s}$ and the $\mathrm{P}$ velocity is set to be $v_{p}=\sqrt{3} v_{s}$. Eighteen vertical-component shots are distributed on the free surface which are marked as the red stars in Figure 3a. The peak frequency of the source wavelet is $30 \mathrm{~Hz}$. There are two levels of parallelization, one for the sources and one for domain decomposition, and the total recording time is $0.32 \mathrm{~s}$ with a $0.08 \mathrm{~ms}$ time step.

The observed dispersion curve is first picked from the spectrum computed by the Radon 
transform in the frequency domain. Each trace of the CSGs is compensated for attenuation and only the traces with their offsets less than $80 \mathrm{~m}$ are used. The fundamental dispersion curves for each CSG are picked along the dominant azimuths from $0^{\circ}$ to $360^{\circ}$ with an interval of $5^{\circ}$. For example, Figure 8 shows the observed dispersion curves from the CSGs with their sources located at points A and B indicated in Figure 3a, where the black dashed lines represent the contours of the observed dispersion curves. The cyan dash-dot lines in Figure 8 represent the contours of the initial dispersion curves.

3D TWD is then used to invert the picked dispersion curves for the S-velocity tomogram. Figure $6 \mathrm{~b}$ displays the inverted S-wave velocity model after 15 iterations, and its associated vertical slices at $y=80 \mathrm{~m}$ and $160 \mathrm{~m}$ are shown in Figures $7 \mathrm{~b}$ and $7 \mathrm{~d}$, respectively; these results agree well with the true model. The contours of the predicted dispersion curves for the sources located at points A and B are represented by the red lines in Figure 8, which correlate well with the contours of the observed dispersion curves.

[Figure 6 about here.]

[Figure 7 about here.]

[Figure 8 about here.]

\section{D Foothills Model}

The topography of the 3D Foothills model shown in Figure 9 is extracted from the 3D SEG Advanced Modeling (SEAM) phase II foothills model (Oristaglio, 2012), where the red lines are the geodesic paths on the triangular mesh for the source marked as the red star. The maximum elevation difference of the topography is $1.2 \mathrm{~km}$. The $3 \mathrm{D}$ Foothills S-wave 
velocity model shown in Figure 10a is modified from the 2D Foothills model in Figure 2a of Brenders et al. (2008). The P-wave velocity is defined as $v_{p}=\sqrt{3} v_{s}$ and the physical size of the velocity model is $7 \mathrm{~km}$ and $3.5 \mathrm{~km}$ in the $\mathrm{x}$ and $\mathrm{y}$ directions, respectively, and is $2 \mathrm{~km}$ deep in the z-direction. The mesh used in the SPECFEM3D is shown in Figure 10b. The initial S-velocity model is shown in Figure 10c. Figure 11 shows the acquisition geometry for this experiment, where 2312 geophones are distributed on the surface, which are arranged in 17 parallel lines along the $\mathrm{x}$-direction, and each line has 136 receivers. The in-line and crossline receiver intervals are $50 \mathrm{~m}$ and $190 \mathrm{~m}$, respectively. There are 80 vertical-component shots distributed on a $10 \times 8$ grid with source intervals of $750 \mathrm{~m}$ and $380 \mathrm{~m}$ in the $\mathrm{x}$ and $\mathrm{y}$ directions, respectively. The peak frequency of the source is $5 \mathrm{~Hz}$ and the observed data are recorded for 2.40 seconds with a $0.8 \mathrm{~ms}$ sampling rate.

The fundamental dispersion curves for each CSG are picked for the frequencies from 2 to $9 \mathrm{~Hz}$ along the dominant azimuths from $0^{\circ}$ to $360^{\circ}$ with an interval of $5^{\circ}$. For example, Figure 12 shows the observed dispersion curves calculated from the CSGs for the sources located at points A, B, C and D indicated in Figure 11, where the black dashed lines represent the contours of the observed dispersion curves. The cyan lines represent the contours of initial dispersion curves.

3D TWD is then used to invert for the S-velocity tomograms. Figure 10d displays the inverted S-wave velocity model. The vertical slices for the true, initial and inverted models are shown in Figures 13a, 13b and 13c, respectively, where the black- and white- dashed lines indicate the large velocity contrast boundaries and the boundaries $0.5 \mathrm{~km}$ below the free surface, respectively. The depth slices $300 \mathrm{~m}$ below the free surface for the true, initial and inverted models are shown in Figures 14a, 14b and 14c, respectively. We can see that the S-velocity model is significantly updated in the shallow part, where most updates are 
confined to the region within $0.5 \mathrm{~km}$ from the surface. The overall velocity structure is well recovered, even though some small-scale features are still missing, which might be caused by the limited frequency content in the data.

The contours of the predicted dispersion curves for the sources located at points A, B, $\mathrm{C}$ and $\mathrm{D}$ in Figure 11 are represented by the red dash-dot lines in Figure 12, which agree well with the contours of the observed dispersion curves. Figure 15 compares the observed (red) and synthetic (blue) traces at the far source-receiver offsets predicted from the initial and inverted models for (a) and (b) with the CSG at B, and (c) and (d) with the CSG at C. Figure 16 shows the common offset gathers (COGs) with offset $2.85 \mathrm{~km}$, which are retrieved from the traces located at the green rectangles in Figure 11 of the CSGs with the sources located at the green stars in Figure 11. Here the red and blue wiggles represent the observed and predicted COGs, respectively. It can be seen that the synthetic waveforms computed from the 3D TWD tomogram closely agree with the observed ones.

[Figure 9 about here.]

[Figure 10 about here.]

[Figure 11 about here.]

[Figure 12 about here.]

[Figure 13 about here.]

[Figure 14 about here.]

[Figure 15 about here.] 
[Figure 16 about here.]

\section{Washington Fault Seismic Data}

A 3D seismic survey was conducted across the Washington fault zone of northern Arizona in 2008 (Figure 17a) and then the Utah and Arizona Geological Surveys (UGS) excavated three trenches over that area (Figure 17b). The 3D acquisition geometry consists of six parallel lines and each line has 80 receivers with a $1 \mathrm{~m}$ spacing near the fault scarp and a 2 $\mathrm{m}$ spacing far away from the fault scarp. The length of each line is $119 \mathrm{~m}$ and the cross-line spacing is $1.5 \mathrm{~m}$. The seismic source is a 10-lb sledgehammer striking a metal plate on the ground. Shots are activated at every other geophone and the experiment geometry is shown in Figure 18. One of the CSGs (\# 87) is shown in Figure 19, where the observed data are recorded for 0.5 seconds with a $0.25 \mathrm{~ms}$ sampling rate.

[Figure 17 about here.]

[Figure 18 about here.]

[Figure 19 about here.]

The 3D data set was impacted by an unpredictable time delay between the source initiation time and the onset of the data recording. This issue was identified by non-zero amplitudes at the zero time for the near-offset trace. To correct this hardware error, the traces in the shot gather of the $3 \mathrm{D}$ data set were advanced by a constant time value $\tilde{t}(\boldsymbol{s})$. To correct for this timing error, the timing error $\tilde{t}(s)$ is obtained by minimizing the summation 
of the picked traveltime differences,

$$
\tilde{t}(\boldsymbol{s})=\underset{\tilde{t}(\boldsymbol{s})}{\arg \min } \sum_{i}\left(\tilde{t}(\boldsymbol{s})+t\left(\boldsymbol{g}_{i}, \boldsymbol{s}\right)-t\left(\boldsymbol{s}, \boldsymbol{g}_{i}\right)\right)^{2},
$$

where $t\left(\boldsymbol{g}_{i}, \boldsymbol{s}\right)$ is the traveltime picked from the trace located at $\boldsymbol{g}_{i}$ of the CSG with the source located at $s$. Figure 20 shows the picked traveltime matrices of traveltime picks for all the shot gathers on line \#4 before and after correction. After correction, the picked traveltimes are more continuous in the common receiver gathers. Continuity in the arrival times is more important than absolute times in order to compute the correct moveout velocity of the surface waves.

[Figure 20 about here.]

The data are processed before the calculation of the dispersion images. Each trace is normalized to compensate for the effects of attenuation and geometrical spreading. We only include the fundamental-mode Rayleigh waves in the CSG by a muting window. For each shot gather, only receivers within the distance $r_{1}=35 \mathrm{~m}$ from the source are used to calculate the dispersion curves in order to make sure that TWD has sufficient depth penetration and lateral resolution (Liu et al., 2019). The dominant azimuth angles defined in Liu et al. (2019) for most of the CSGs are approximately $0^{\circ}$ and $180^{\circ}$ because of the narrow acquisition geometry. Thus, the fundamental dispersion values are calculated along the azimuthal angles $0^{\circ}$ and $180^{\circ}$. The frequency range in the inversion is from $20 \mathrm{~Hz}$ to 60 $\mathrm{Hz}$, which is the dominant frequency range of surface waves in the data. Figures 21a and 21b show the picked dispersion values for CSGs along line \#4 with the azimuthal angles $\theta=0^{\circ}$ and $\theta=180^{\circ}$, respectively; here, the black dashed lines denote the contours of the observed dispersion curves. At certain frequency ranges, it is difficult to pick the dispersion 
curves because of the low signal-to-noise ratio of the data so that some dispersion curves are missing.

We first approximate an initial model from the picked dispersion curves, which is called a "pseudo 1D S-velocity model" in Liu et al. (2019). That is, the depth $z$ and S-wave velocity $v_{s}$ of the initial model are calculated by scaling the wavelength $\lambda$ and phase velocity $c$ with factors of 0.5 and 1.1 , respectively. The $1 \mathrm{D}$ depth profile is assumed to be centered at the middle of the receiver spread. The 1D velocity profiles are interpolated as the starting model for 2D WD. For example, Figure 22a displays the pseudo 1D S-velocity model beneath line \#4 which is calculated from the dispersion curves in Figure 21. For comparison with the WD tomogram, we interpolated the $1 \mathrm{D}$ velocity profiles from all six lines as the 3D model shown in Figure 23a.

Then, we apply 2D TWD to invert for the $2 \mathrm{D}$ velocity model along the 6 lines. Figures $22 \mathrm{a}$ and $22 \mathrm{~b}$ show the initial and inverted S-velocity models beneath the fourth line, where the white lines indicate the boundaries $10 \mathrm{~m}$ below the free surface. The cyan dash-dot lines in Figure 21 represent the contours of the initial dispersion curves. The contours of the predicted dispersion curves are represented by the red lines in Figure 21, which more closely agree with the contours of the observed dispersion curves, especially for the high frequencies ranging between $45 \mathrm{~Hz}$ to $60 \mathrm{~Hz}$. The 6 inverted 2D S-velocity models are then interpolated to obtain an initial velocity m (see Figure 23b) for 3D TWD. The inverted 3D TWD tomogram is shown-Figure 23c. The 3D TWD cannot make any further update for the 2D TWD tomogram. So the 3D TWD tomogram is almost the same as the initial S-velocity model-and indicates that 2D TWD is sufficient for this dataset because of the narrow acquisition geometry.

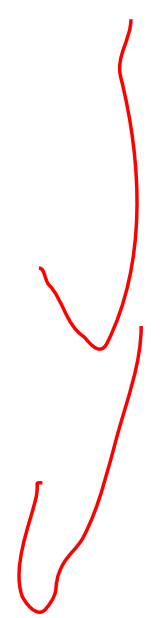


To further test the accuracy of the TWD tomogram, Figure 24 shows the comparison between the observed (blue) and synthetic (red) traces predicted from the (a) initial and (b) inverted S-velocity models for CSGs No. 128. Here, two matched filters are calculated from trace No. 141 at $X=40 \mathrm{~m}$ in Figure 24, respectively. The matched filters are then applied to reshape the synthetic waveform. We can see that the predicted fundamentalmode surface waves closely match the observed ones, especially at the far offset locations $(X>40 \mathrm{~m})$. Figure 25 shows the COGs with the offset of $16 \mathrm{~m}$ for CSGs in line No. 4, where the blue and red wiggles represent the observed and predicted COGs, respectively. The predicted COG is more consistent with the raw data compared to the initial COG.

For comparison, we calculate the 2D P-velocity tomogram shown in Figure 22c by the raypath traveltime inversion method. The $\mathrm{Vp} / \mathrm{Vs}$ ratio map is then calculated and displayed in Figure 22d. From the inverted S-velocity tomogram shown in Figure 22b, we can see there is a low-velocity zone (LVZ) between $\mathrm{X}=40 \mathrm{~m}$ and $\mathrm{X}=80 \mathrm{~m}$. The $\mathrm{LVZ}$ appears in the $\mathrm{P}$ velocity tomogram but the boundaries of the LVZ are ambiguous compared to those shown in the S-velocity tomogram. The LVZ is also clearly shown in the Vp/Vs ratio tomogram, which has a high $\mathrm{Vp} / \mathrm{Vs}$ ratio. The fault scarp at $\mathrm{X}=43 \mathrm{~m}$ ( see Figure 18) is located at the left-hand side of the LVZ. So, we interpret the line labeled with "F1" as the location of the main fault and the LVZ is interpreted as a colluvial wedge labeled with "CW". In Figure 22, the black lines labeled with "F2" are the locations of the interpreted antithetic fault and there is also a possible fault labeled with "F3". The locations of these faults and the S-velocity tomogram are superposed on the COGs in Figure 26. The fault structures appear to be consistent with those seen in the COG image.

The trench was excavated to explore the fault zone by UGS in the spring of 2009 (Hanafy et al., 2015). The trench is across the fault scarp and its location is indicated by the black 
rectangles in Figure 22. The zoom view of the S-velocity and P-velocity tomograms and the $\mathrm{Vp} /$ Vs ratio tomogram in the black rectangles are shown in Figures 27a, 27b and 27c, respectively. The trench log is displayed in Figure $27 \mathrm{~d}$ and exposes a more complex main fault zone (F1). The locations of the main fault in S-velocity tomogram and Vp/Vs ratio tomogram are consistent with those in the trench log. Compared to the 3-D P-wave velocity tomogram, the 3D S-wave tomogram agrees much more closely with the geological model taken from the trench log. The agreement with the trench log is even better when the $\mathrm{Vp} / \mathrm{Vs}$ tomogram is computed, which reveals a sharp change in velocity across the fault. The localized velocity anomaly in the $\mathrm{Vp} / \mathrm{Vs}$ tomogram is in very good agreement with the well log. Our results suggest that integrating the Vp and Vs tomograms can sometimes give the most accurate estimates of the subsurface geology across normal faults.

[Figure 21 about here.]

[Figure 22 about here.]

[Figure 23 about here.]

[Figure 24 about here.]

[Figure 25 about here.]

[Figure 26 about here.]

[Figure 27 about here.] 


\section{DISCUSSION}

Irregular topography has a significant impact on surface-wave propagation, which distorts seismic wavefronts by strong scattering and attenuation in a complex manner. When the wavelength is smaller than the characteristic wavelength of the topographic relief, the source-receiver distance factor may play a significant role for calculating the phase velocity of surface waves, which is essential for 3D TWD and discussed by Li et al. (2019b). Failure to use the actual source-receiver distance in the evaluation of the phase velocity can lead to errors in the inverted model for 3D TWD. Results with the homogeneous model suggest that there will be significant errors in the dispersion curve without consideration of the topography. By considering the topography properly, we can get a more accurate dispersion curve as shown in the Foothills examples.

The elevation changes in our 3D Foothill example are between two and four S-wave wavelengths. Accurate wavefield modeling using a 3D elastic SEM is an important ingredient for successful 3D TWD inversions in such a challenging geologic setting. As shown in Figure 13, the S-velocity model is significantly updated only in the shallow part (about $0.5 \mathrm{~km}$ deep from the surface). This is reasonable because the maximum wavelength of the surface waves is $1.0 \mathrm{~km}$ approximately and surface waves are typically most sensitive to the velocity model to a depth of approximately one-half of their wavelength (Liu et al., 2017; Hyslop and Stewart, 2015).

Our field data example shows no significant improvement for the 3D TWD results compared to the $2 \mathrm{D}$ results. This is because of the relatively narrow recording geometry of the Arizona survey. Considering the high computational cost for 3D TWD, 3D TWD might be too costly and not significantly beneficial compared to 2D TWD for some field surveys. 
However, the improvement of 3D WD in accuracy compared to 2D WD can sometimes make this extra cost worthwhile when there are significant near-surface lateral variations in the S-velocity distribution (Liu et al., 2019).

In the field data test, the predicted and observed dispersion contours at low frequency range in Figure 21 do not match as good as those in the synthetic tests, for example the dispersion contours in Figure 12. This may be because of the uncertainties when picking the observed dispersion curves and the uncertainties increase in the low frequency range or with a small number of receivers within a specified offset range (Foti et al. 2018). To reduce the picking uncertainties, high-resolution surface wave dispersion imaging method is needed (Krohn and Routh, 2016).

High Vp/Vs ratios in the LVZ of the tomograms (Figure 22d) might be caused by the groundwater saturation in the fault zone. Groundwater saturation has a major effect on Pwave velocities in the near surface, where saturated materials typically have higher P-wave velocities than unsaturated or partially saturated materials due to the higher incompressibility (e.g., bulk modulus, K) of the saturated materials. However, S-wave velocities are much less affected by groundwater saturation because $\mathrm{S}$ waves do not include a bulk modulus term (Catchings et al., 2014). When the sediment in the LVZ is saturated with groundwater, the P-wave velocity is increased so that the boundaries of the LVZ are ambiguous in the P-velocity tomogram. However, the S-wave velocity is much less affected so that the boundaries of the LVZ are more clearly delineated in the S-velocity tomogram. 


\section{CONCLUSIONS}

We extend the $2 \mathrm{D}$ TWD methodology to $3 \mathrm{D}$, that accounts for significant $3 \mathrm{D}$ variations in topography by a 3D spectral element solver. The objective function of 3D TWD is the sum of the squared differences between the predicted and observed dispersion curves. More accurate dispersion curves can be calculated by using the geodesic distance compared to that using the Euclidean distance, which can lead to a more accurate inverted model for 3D TWD. The effectiveness of this method is numerically demonstrated with synthetic and field data recorded on an irregular free surface. Results with synthetic data suggest that 3D TWD can accurately invert for the S-velocity model in the Foothills region when there is a huge elevation difference compared to the $\mathrm{S}$-wave wavelengths. Compared to the 3-D P-wave velocity tomogram, the 3D S-wave tomogram agrees much more closely with the geological model taken from the trench log. The agreement with the trench log is even better when the $\mathrm{Vp} / \mathrm{Vs}$ tomogram is computed, which reveals a sharp change in velocity across the fault that is in very good agreement with the well log. Our results suggest that integrating the Vp and Vs tomograms can sometimes give the most accurate estimates of the subsurface geology across normal faults.

Similar to 3D WD, a limitation of 3D TWD is that the fundamental dispersion curves must be picked for each shot gather. This process can be prone to errors when there is a strong overlap with higher-order modes or there is spatial and temporal aliasing due to large spatial and temporal sampling intervals. This problem might be mitigated by the machine learning method that automatically picks dispersion curves. 


\section{ACKNOWLEDGEMENTS}

We dedicate this paper to Dimitri Komatitsch and his loving family members, he is a light that has gone out much too early. We also thank Ron Bruhn for his valuable advice about the geological explanation of our field results. The research reported in this publication was supported by the King Abdullah University of Science and Technology (KAUST) in Thuwal, Saudi Arabia. We are grateful to the sponsors of the Center for Subsurface Imaging and Modeling Consortium for their financial support. For computer time, this research used the

resources of the Supercomputing Laboratory at KAUST and the IT Research Computing Group. We thank them for providing the computational resources required for carrying out this work. 


\section{REFERENCES}

Borisov, D., R. Modrak, F. Gao, and J. Tromp, 2018, 3D elastic full-waveform inversion of surface waves in the presence of irregular topography using an envelope-based misfit function: Geophysics, 83, no. 1, R1-R11.

Brenders, A., S. Charles, and R. Pratt, 2008, Velocity estimation by waveform tomography in the Canadian Foothill-a synthetic benchmark study: Presented at the 70th EAGE Conference and Exhibition incorporating SPE EUROPEC 2008.

Catchings, R. D., M. J. Rymer, M. R. Goldman, R. R. Sickler, and C. J. Criley, 2014, A method and example of seismically imaging near-surface fault zones in geologically complex areas using vp, vs, and their ratios: Bulletin of the Seismological Society of America, 104, no. 4, 1989-2006.

Chen, J., and Y. Han, 1990, Shortest paths on a polyhedron: Proceedings of the sixth annual symposium on Computational geometry, ACM, 360-369.

Dijkstra, E. W., 1959, A note on two problems in connexion with graphs: Numerische Mathematik, 1, no. 1, 269-271.

Dou, S., and J. B. Ajo-Franklin, 2014, Full-wavefield inversion of surface waves for mapping embedded low-velocity zones in permafrost: Geophysics, 79, no. 6, EN107-EN124.

Eguiluz, A. G., and A. A. Maradudin, 1983, Frequency shift and attenuation length of a Rayleigh wave due to surface roughness: Phys. Rev. B, 28, 728-747.

Foti, S., F. Hollender, F. Garofalo, D. Albarello, M. Asten, P.-Y. Bard, C. Comina, C. Cornou, B. Cox, G. Di Giulio, et al., 2018, Guidelines for the good practice of surface wave analysis: A product of the interpacific project: Bulletin of Earthquake Engineering, 16, no. $6,2367-2420$.

Fu, L.-Y., and R.-S. Wu, 2001, A hybrid BE-GS method for modeling regional wave prop- 
agation: Pure Appl. Geophys., 158, no. 7, 1251-1277.

Groos, L., M. Schäfer, T. Forbriger, and T. Bohlen, 2014, The role of attenuation in 2D full-waveform inversion of shallow-seismic body and Rayleigh waves: Geophysics, 79, no. 6, R247-R261.

— 2017 , Application of a complete workflow for 2D elastic full-waveform inversion to recorded shallow-seismic Rayleigh waves: Geophysics, 82(2), R109-R117.

Hanafy, S. M., A. Mattson, R. L. Bruhn, S. Liu, and G. T. Schuster, 2015, Outcrops and well logs as a practicum for calibrating the accuracy of traveltime tomograms: Interpretation, 3, no. 3, SY27-SY40.

Haskell, N. A., 1953, The dispersion of surface waves on multilayered media: Bull. Seismol. Soc. Am., 43, no. 1, 17-34.

Hyslop, C., and R. R. Stewart, 2015, Imaging lateral heterogeneity using reflected surface waves: Geophysics, 80, no. 3, EN69-EN82.

Köhler, A., C. Weidle, and V. Maupin, 2012, On the effect of topography on surface wave propagation in the ambient noise frequency range: J. Seismolog., 16, no. 2, 221-231.

Komatitsch, D., and J. Tromp, 1999, Introduction to the spectral element method for threedimensional seismic wave propagation: Geophys. J. Int., 139, no. 3, 806-822.

Komatitsch, D., and J.-P. Vilotte, 1998, The spectral element method: An efficient tool to simulate the seismic response of 2D and 3D geological structures: Bull. Seismol. Soc. Am., 88, no. 2, 368 .

Krohn, C. E., and P. S. Routh, 2016, Exploiting surface consistency for surface-wave characterization and mitigationpart 2: Application to 3D data: Geophysics, 82, no. 1, V39-V50. Li, J., Z. Feng, and G. Schuster, 2017a, Wave-equation dispersion inversion: Geophys. J. Int., 208, 1567-1578. 
Li, J., S. Hanafy, Z. Liu, and G. T. Schuster, 2019a, Wave equation dispersion inversion of love waves: Geophysics, 84, no. 5, 1-45.

Li, J., F.-C. Lin, A. Allam, Y. Ben-Zion, Z. Liu, and G. Schuster, 2019b, Wave equation dispersion inversion of surface waves recorded on irregular topography: Geophys. J. Int., 217, no. 1, 346-360.

Li, J., and G. Schuster, 2016, Skeletonized wave equation of surface wave dispersion inversion: SEG Technical Program Expanded Abstracts 2016, Society of Exploration Geophysicists, Society of Exploration Geophysicists, 3630-3635.

Li, J., G. T. Schuster, F.-C. Lin, and A. Alam, 2017b, Wave-equation dispersion inversion of surface waves recorded on irregular topography: SEG Technical Program Expanded Abstracts 2017, 2621-2626.

Liu, Z., A. AlTheyab, S. M. Hanafy, and G. Schuster, 2017, Imaging near-surface heterogeneities by natural migration of backscattered surface waves: Field data test: Geophysics, 82(3), S197-S205.

Liu, Z., and L. Huang, 2019, Multiscale and layer-stripping wave-equation dispersion inversion of Rayleigh waves: Geophys. J. Int., 218, no. 3, $1807-1821$.

Liu, Z., J. Li, S. M. Hanafy, and G. T. Schuster, 2018, 3D wave-equation dispersion inversion of surface waves: SEG Technical Program Expanded Abstracts 2018, 4733-4737.

- 2019, 3D wave-equation dispersion inversion of Rayleigh waves: Geophysics, 84, no. $5,1-127$.

Mayer, A. P., W. Zierau, and A. A. Maradudin, 1991, Surface acoustic waves propagating along the grooves of a periodic grating: J. Appl. Phys., 69, no. 4, 1942-1947.

Mitchell, J. S., D. M. Mount, and C. H. Papadimitriou, 1987, The discrete geodesic problem: SIAM J. Comput., 16, no. 4, 647-668. 
Modrak, R. T., D. Borisov, M. Lefebvre, and J. Tromp, 2018, Seisflows-flexible waveform inversion software: Computers \& Geosciences, 115, 88 - 95 .

Nocedal, J., and S. Wright, 2006, Numerical optimization: Springer Science \& Business Media.

Nuber, A., E. Manukyan, and H. Maurer, 2016, Ground topography effects on near-surface elastic full waveform inversion: Geophys. J. Int., 207, no. 1, 67-71.

Oristaglio, M., 2012, Seam update: The Leading Edge, 31, no. 10, 1130-1132.

O'Rourke, J., 1999, Computational geometry column 35: SIGACT News, 30, no. 2, 31-32.

Pan, Y., L. Gao, and T. Bohlen, 2018, Time-domain full-waveform inversion of Rayleigh and Love waves in presence of free-surface topography: J. Appl. Geophys., 152, $77-85$.

Park, C. B., R. D. Miller, and J. Xia, 1999, Multichannel analysis of surface waves: Geophysics, 64, no. 3, 800-808.

Pérez Solano, C. A., D. Donno, and H. Chauris, 2014, Alternative waveform inversion for surface wave analysis in 2-D media: Geophys. J. Int., 198, no. 3, 1359-1372.

Plessix, R.-E., and W. Mulder, 2004, Frequency-domain finite-difference amplitudepreserving migration: Geophys. J. Int., 157, no. 3, 975-987.

Sethian, J., 2001, Evolution, implementation, and application of level set and fast marching methods for advancing fronts: J. Comput. Phys., 169, no. 2, $503-555$.

Sethian, J. A., and A. M. Popovici, 1999, 3-D traveltime computation using the fast marching method: Geophysics, 64, no. 2, 516-523.

Snieder, R., 1986, The influence of topography on the propagation and scattering of surface waves: Phys. Earth Planet. Inter., 44, no. 3, 226-241.

Surazhsky, V., T. Surazhsky, D. Kirsanov, S. J. Gortler, and H. Hoppe, 2005, Fast exact and approximate geodesics on meshes: ACM transactions on graphics (TOG), ACM, 
553-560.

Xia, J., R. Miller, C. Park, and G. Tian, 2002, Determining Q of near-surface materials from Rayleigh waves: J. Appl. Geophys., 51, no. 2-4, 121-129.

Xia, J., R. D. Miller, and C. B. Park, 1999, Estimation of near-surface shear-wave velocity by inversion of Rayleigh waves: Geophysics, 64, no. 3, 691-700.

Yuan, Y. O., F. J. Simons, and E. Bozdă̆, 2015, Multiscale adjoint waveform tomography for surface and body waves: Geophysics, 80, no. 5, R281-R302.

\section{APPENDIX A}

\section{CALCULATION OF THE GEODESIC}

P

A Iratural shortest paths problem with many applications is the following: given two points $s$ and $r$ on the surface of a polyhedron of $n$ vertices, find the shortest path on the surface from $s$ to $r$. This type of within-surface shortest path is often called a geodesic shortest path, in contrast to a Euclidean shortest path (O’Rourke, 1999).

The computation of geodesic paths is a common operation in many computer graphics applications (Surazhsky et al., 2005) and the computation of seismic travel times (Sethian and Popovici, 1999). There are many methods for computing the geodesic distance on the topography represented by the triangle mesh. Most of the algorithms bear a very close resemblance to the famous Dijkstra algorithm (Dijkstra, 1959) that finds the shortest paths on graphs, for example, the fast marching method (Sethian, 2001) and the exact geodesic algorithm (Mitchell et al., 1987). The exact geodesic algorithm uses the continuous Dijkstra method and simulates the continuous propagation of a wavefront of points equidistant from $s$ across the surface. The method has $O\left(n^{2} \log n\right)$ worst-case time complexity, but in practice 
can work with million-node meshes in a reasonable time. An exact geodesic algorithm with worst-case time complexity of $O\left(n^{2}\right)$ was described by Chen and Han (1990).

The most straightforward explanation of the exact geodesic algorithm is unfolding. If we want to find the shortest path on the surface of a nple surface mesh shown in Figure 28, we can find a sequence of edge-adjacent faces $f_{1}, f_{2}, \cdots, f_{7}$ and unfold face $f_{i+1}$ onto the plane of $f_{i}$ as shown in Figure $28 \mathrm{~b}$ so that the shortest path is the straight line. The detailed implementation can be referred to Surazhsky et al. (2005) and Chen and Han (1990). In this paper, we firstly generate a triangular mesh for the topography by CUBIT. Then, we compute the geodesic distance using the exact geodesic algorithm implemented by Surazhsky et al. (2005).

[Figure 28 about here.]

\section{APPENDIX B}

\section{DISCRETE RADON TRANSFORM}

Assume that $\tilde{\boldsymbol{g}}=r_{g}(\cos \theta, \sin \theta, 0)$ is the mapping point from the receiver $\boldsymbol{g}=\left(x_{g}, y_{g}, z_{g}\right)$
to plane $z=0$, where $r_{g}$ and $\theta$ are the geodesic distance and azimuth angle, respectively. So, the domain of the data $d(\boldsymbol{g}, t)$ is changed to $(\tilde{\boldsymbol{g}}, t)$. The discrete Radon transform of the shot gather $d(\tilde{\boldsymbol{g}}, t)$ is

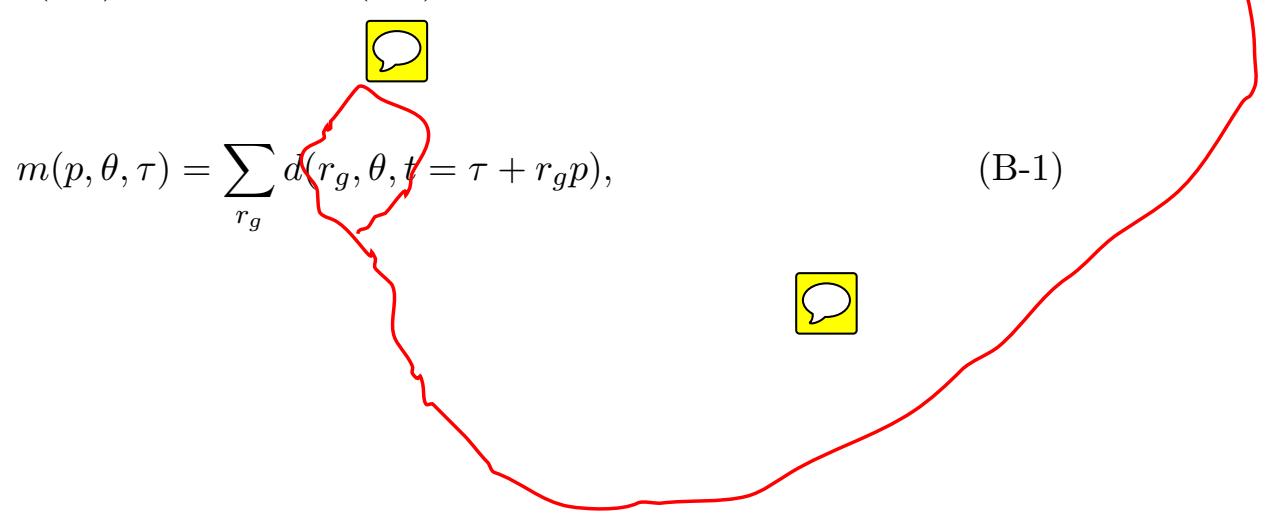


where $\boldsymbol{p}=p(\cos \theta, \sin \theta)$ is the slowness vector, and $p$ is the slowness value along the aizmuth angle $\theta$. Apply a Fourier transform to equation B-1 gives

$$
\begin{aligned}
\tilde{m}(p, \theta, \omega) & =\sum_{r_{g}} \int_{-\infty}^{\infty} d\left(r_{g}, \theta, \tau+r_{g} p\right) e^{i \omega \tau} d \omega, \\
& =\sum_{r_{g}}\left[\int_{-\infty}^{\infty} d\left(r_{g}, \theta, \tau\right) e^{i \omega \tau} d \omega\right] e^{-i \omega p r_{g}}, \\
& =\sum_{r_{g}} \tilde{d}\left(r_{g}, \theta, \omega\right) e^{-i \omega p r_{g}},
\end{aligned}
$$

where $\tilde{d}\left(r_{g}, \theta, \omega\right)$ is the Fourier spectrum of the data $d\left(r_{g}, \theta, t\right)$, and $\tilde{m}(p, \theta, \omega)$ is the Fourier spectrum of the Radon-transformed data $m(p, \theta, \tau)$. The fundamental-mode dispersion curve for the azimuth $\theta, C(\omega, \theta)$, is picked from the magnitude spectrum of $\tilde{m}(p, \theta, \omega)$. 


\section{LIST OF FIGURES}

1 Schematic diagram shows the offset distance $l$ along the (a) flat and (b) irregular surfaces from the source at $\mathbf{s}$ (the red star) to the receiver at $\mathbf{r}_{\mathbf{1}}$, where $l_{e}$ is the Euclidean distance. . . . . . . . . . . . .

2 Schematic diagram shows the offset $L$ and the azimuth $\theta$ from the source at $\mathbf{s}$ (red star) to the receiver at $\mathbf{r} . \ldots \ldots \ldots$

3 (a) Acquisition geometry where the yellow area shows the locations of the receivers (black asterisks) within the azimuth angle ranged from $277.5^{\circ}$ to $282.5^{\circ}$ for the source at $\mathrm{A}$, where the source is represented by the red star; (b) paths of the geodesics on the topography from the source at $A$ to the receivers that are marked as the black asterisks in (a); (c) differences between the geodesic and Euclidean distances, where the trace number is numbered according to the geodesic distance in ascending order; (d) CSG for trace No. 1 to 30 from the model with (red) and without (blue) topography. . . . . .

4 Dispersion image calculated by the (a) Euclidean and (b) geodesic distances for the data recorded in the irregular surface. (c) Dispersion image calculated for the data recorded in the flat surface. Here, the green curves are the theoretical phase velocity dispersion curves $(c=919.4 \mathrm{~m} / \mathrm{s})$ and the red curves are the picked dispersion curves. . . . . . . . . . . . . .

5 Dispersion curves for the data from the flat-surface model and their contours are represented by the black dashed lines. Here, the cyan lines and the red dash-dot lines represent the contours of the dispersion curves calculated by the Euclidean and geodesic distances from the model with the topography, respectively. . . . . . . . . . . . . . . .

6 (a) True S-velocity checkerboard model and (b) S-veocity tomogram by 3D

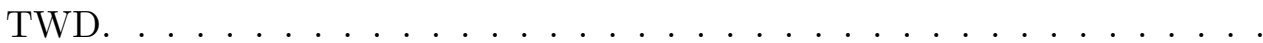

7 True S-velocity slices at $y=$ (a) $80 \mathrm{~m}$ and (c) $160 \mathrm{~m}$. Inverted S-velocity slices at $y=($ b) $80 \mathrm{~m}$ and $(\mathrm{d}) 160 \mathrm{~m} . \ldots \ldots \ldots$

8 Observed dispersion curves from the CSGs with their sources located at points (a) A and (b) B (indicated in Figure 3a), where the black dashed lines, the cyan and red dash-dot lines represent the contours of the observed, initial and inverted dispersion curves, respectively. . . . . . . . . .

9 Topography of the 3D Foothill model, where the red lines are the geodesic paths for the source marked by the red star. . . . . . . . . . . . . .

10 (a) True S-velocity model, (b) corresponding mesh, (c) initial S-velocity model and $(\mathrm{d})$ S-velocity tomogram. . . . . . . . . . . . . .

11 Acquisition geometry for the numerical tests with data generated for the 3D Foothill model, where the red dots and blue circles indicate the locations of the receivers and sources, respectively. . . . . . . . . . . . 
12 Observed dispersion curves for the sources located at (a) A, (b) B, (c) C and (d) D indicated in Figure 11b, where the black dashed lines, the cyan dash-dot lines and the red lines represent the contours of the observed, initial and inverted dispersion curves, respectively. . . . . . . . . . . . .

13 Slices of the (a) true, (b) initial, and (c) inverted S-velocity models at $\mathrm{y}=433$ $\mathrm{m}$, where the black and white dashed lines indicate the large velocity contrast boundaries and the boundaries $0.5 \mathrm{~km}$ below the free surface, respectively. .

14 Depth slices $300 \mathrm{~m}$ below the surface for the (a) true, (b) initial and (c) inverted Foothill S-velocity models, where the black dashed lines indicate the large velocity contrast boundaries. . . . . . . . . . . . . . .

15 Comparison between the observed (red) and synthetic (blue) traces at far offsets predicted from the initial model (LHS panels) and 3D tomogram (RHS panels) for CSG B in (a) and (b), and CSG C in (c) and (d). Here, the locations of points $\mathrm{B}$ and $\mathrm{C}$ and the line numbers are indicated in Figure 11.

16 COGs with the offset of $2.85 \mathrm{~km}$, which are retrieved from the traces located at the green rectangles in Figure 11 of the CSGs with the sources located at the green stars in Figure 11. Here the red and blue wiggles represent the observed and predicted COGs, respectively. . . . . . . . . . . . .

17 (a) Map of the Washington fault and the survey site. The location of the survey site is $5 \mathrm{~km}$ south of the Utah-Arizona border. (b) Topographic map around the seismic survey, where the red and green rectangles indicate the locations of the 3D seismic survey and the trench site, respectively. . . . . .

18 Survey geometry for the 3D experiment in the Washington fault zone. The open red circles denote the locations of sources and the solid blue dots denote the locations of receivers. The dashed black line denotes the location of the fault scarp. . . . . . . . . . . . . . . . .

19 Common shot gather \# 87 of Washington fault data. . . . . . . . . . . .

20 Traveltime matrices before and after the correction of the acquisition hardware error for the $2 \mathrm{D}$ data set on line \#4. . . . . . . . . . . . . .

21 (a) Observed dispersion curves for the CSGs on Line \# 4 along the azimuthal angles (a) $\theta=0^{\circ}$ and (b) $\theta=180^{\circ}$, where the black dashed lines, the cyan dash-dot lines and the red lines represent the contours of the observed, initial and inverted dispersion curves, respectively. . . . . . . . . . . . .

22 (a) Initial and (b) inverted S-wave velocity models beneath line \#4. (c) Pwave velocity tomogram calculated from the picked traveltimes in Figure 20b. (d) $\mathrm{Vp} / \mathrm{Vs}$ ratio tomogram beneath line \#4. Here the white lines indicate the boundaries $10 \mathrm{~m}$ below the free surface. The trench is excavated in the locations of the black rectangles. The lines labeled with "F1" and "F2" are interpreted as the locations of the main fault and the antithetic fault. The line labeled with "F3" is the location of another possible fault. "CW" represents the colluvial wedge. . . . . . . . . . . . 
23 (a) Initial, (b) 2D and (c) 3D S-wave velocity tomograms. Here, the depth and S-wave velocity of the initial model are calculated by scaling the wavelength and phase velocity with factors of 0.5 and 1.1, respectively (Liu et al., 2019). 3D TWD cannot make any further update for the 2D TWD tomogram so that the 3D TWD tomogram in (a) is almost the same as the 2D TWD tomogram in $(\mathrm{b})$. . . . . . . . . . . . . . .

24 Comparison between the observed (blue) and synthetic (red) traces predicted from the (a) initial and (b) inverted S-velocity models for CSG \# 128. . . .

25 COGs with the offset of $16 \mathrm{~m}$ for line \# 4 calculated from the (a) initial and (b) inverted S-velocity models, where the blue and red wiggles represent the observed and predicted COGs, respectively. . . . . . . . . . . . .

26 Observed COGs with the offset of $16 \mathrm{~m}$ are superposed on the S-velocity tomogram, where the COGs are adjusted by following the topography. . .

27 Zoom views of (a) S-velocity and (b) P-velocity tomograms and (c) Vp/Vs tomogram in Figure 22. (d) Ground truth extracted from a nearby trench $\log ($ Hanafy et al., 2015). . . . . . . . . . . . . . . .

28 Schematic diagram of the calculation of the geodesic on a simple surface mesh by unfolding. . . . . . . . . . . . . . . . . . . 


\section{a) Flat Surface}

$$
l=l_{e}
$$

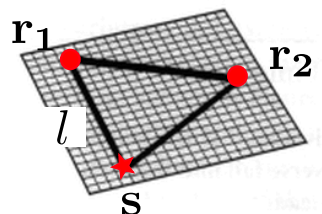

b) Irregular Surface

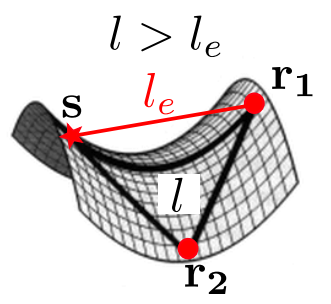

Figure 1: Schematic diagram shows the offset distance $l$ along the (a) flat and (b) irregular surfaces from the source at $\mathbf{s}$ (the red star) to the receiver at $\mathbf{r}_{\mathbf{1}}$, where $l_{e}$ is the Euclidean distance. 


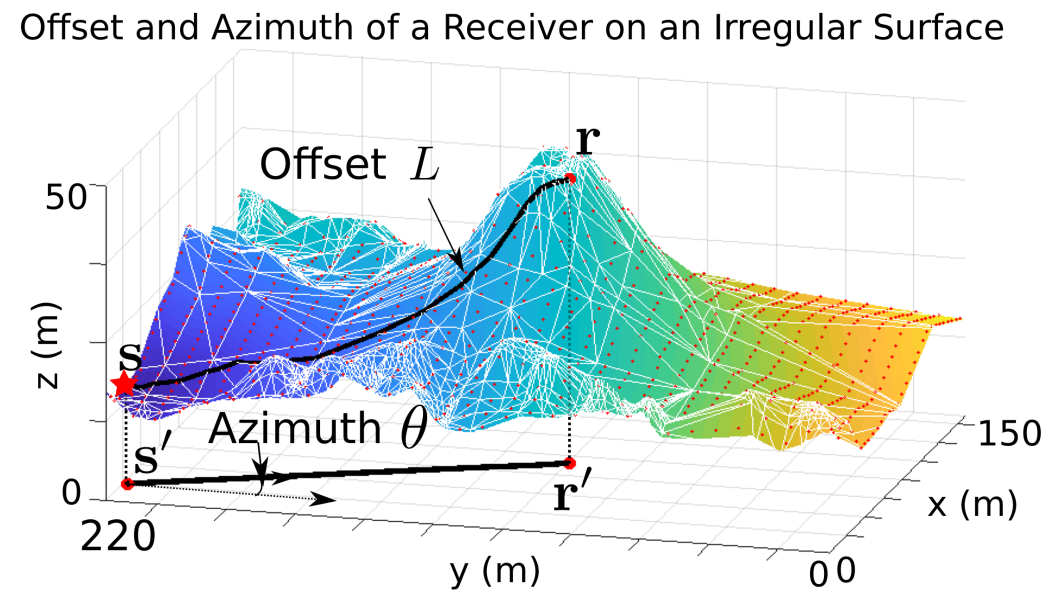

Figure 2: Schematic diagram shows the offset $L$ and the azimuth $\theta$ from the source at $\mathbf{s}$ (red star) to the receiver at $\mathbf{r}$. 
(a) Acquisition Geometry

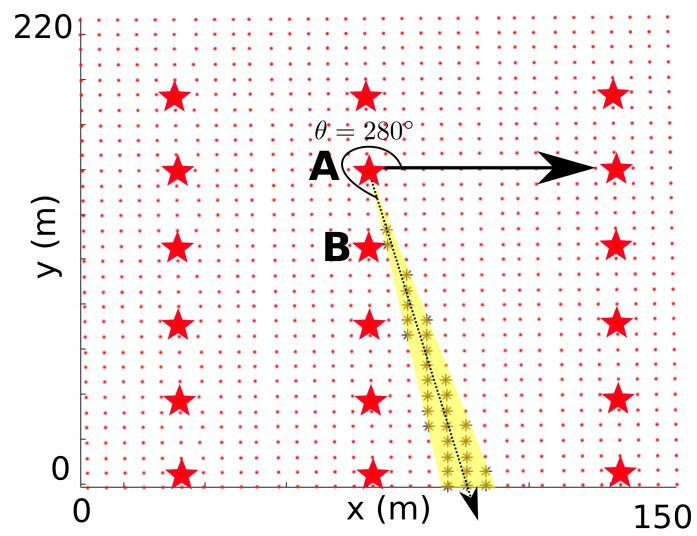

(c) Difference between Geodesic and Euclidean Distances

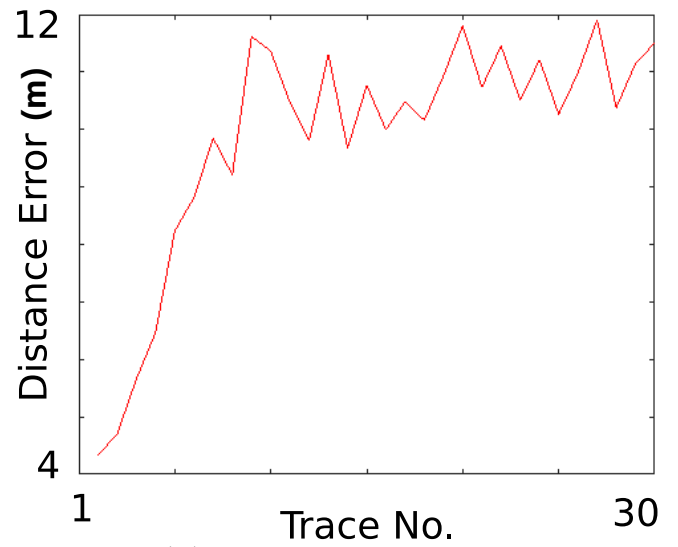

(b) Paths of Geodesic

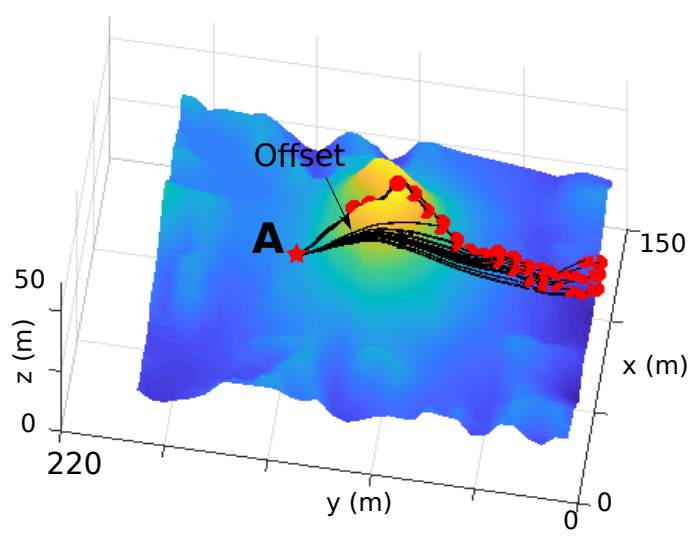

(d) Data with (Red) and without (Blue) Topography

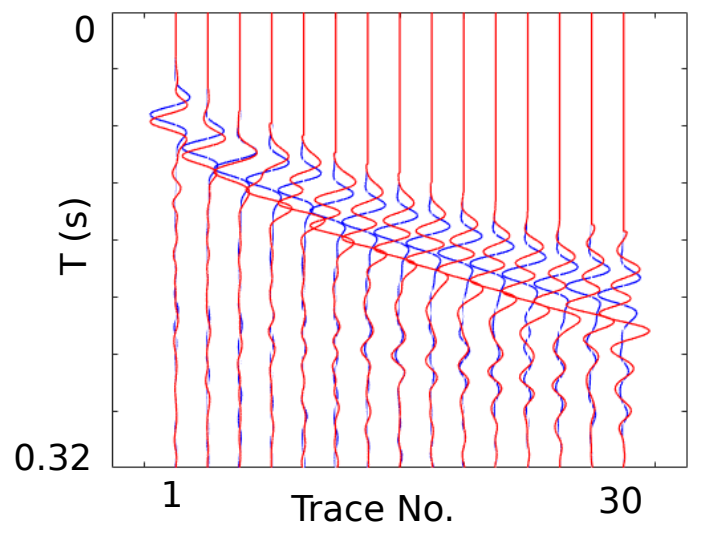

Figure 3: (a) Acquisition geometry where the yellow area shows the locations of the receivers (black asterisks) within the azimuth angle ranged from $277.5^{\circ}$ to $282.5^{\circ}$ for the source at A, where the source is represented by the red star; (b) paths of the geodesics on the topography from the source at $A$ to the receivers that are marked as the black asterisks in (a); (c) differences between the geodesic and Euclidean distances, where the trace number is numbered according to the geodesic distance in ascending order; (d) CSG for trace No. 1 to 30 from the model with (red) and without (blue) topography. 
(a) Euclidean Distance

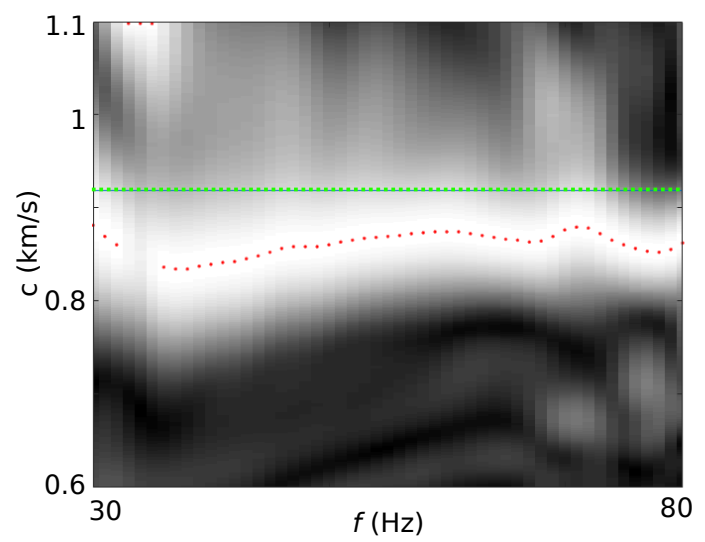

(b) Geodesic Distance

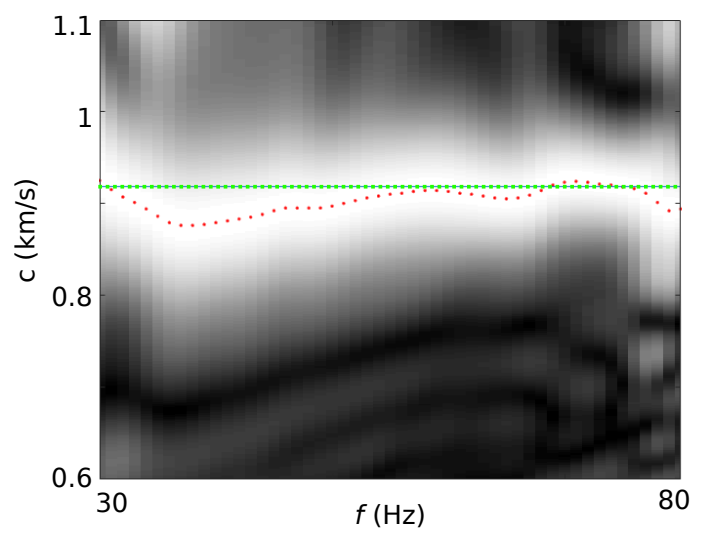

(c) Flat Free Surface

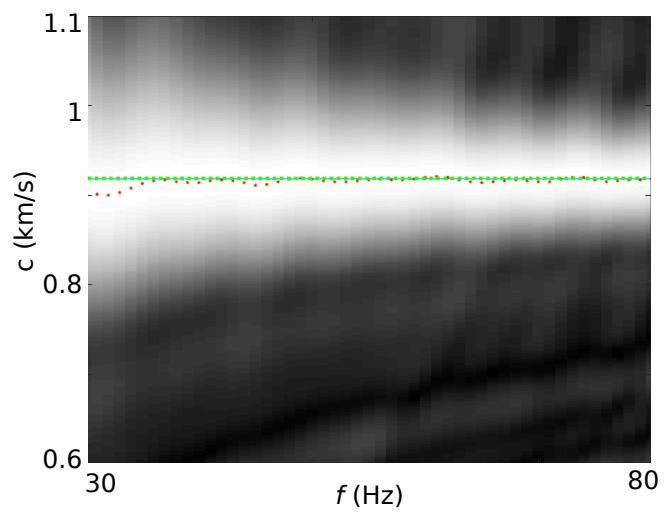

Figure 4: Dispersion image calculated by the (a) Euclidean and (b) geodesic distances for the data recorded in the irregular surface. (c) Dispersion image calculated for the data recorded in the flat surface. Here, the green curves are the theoretical phase velocity dispersion curves $(c=919.4 \mathrm{~m} / \mathrm{s})$ and the red curves are the picked dispersion curves. 


\section{Dispersion Curves}

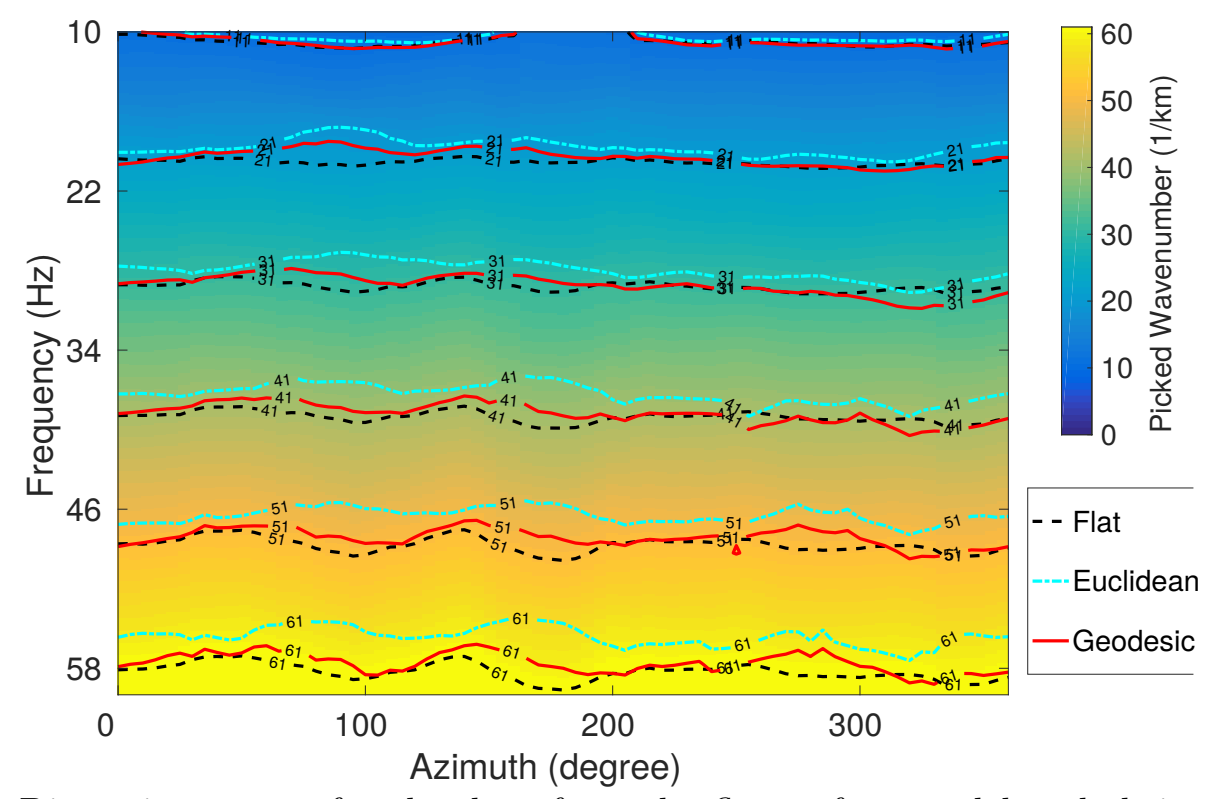

Figure 5: Dispersion curves for the data from the flat-surface model and their contours are represented by the black dashed lines. Here, the cyan lines and the red dash-dot lines represent the contours of the dispersion curves calculated by the Euclidean and geodesic distances from the model with the topography, respectively. 
(a) True S-velocity Model

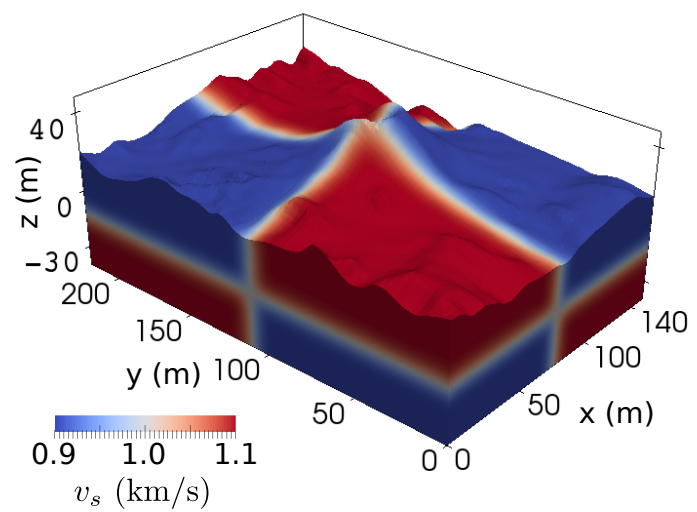

(b) S-velocity Tomogram

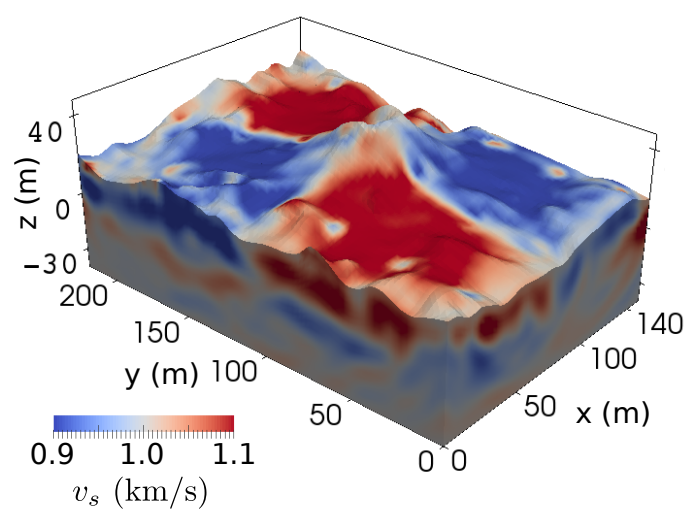

Figure 6: (a) True S-velocity checkerboard model and (b) S-veocity tomogram by 3D TWD. 
(a) True S-velocity Slice at $y=80 \mathrm{~m}$

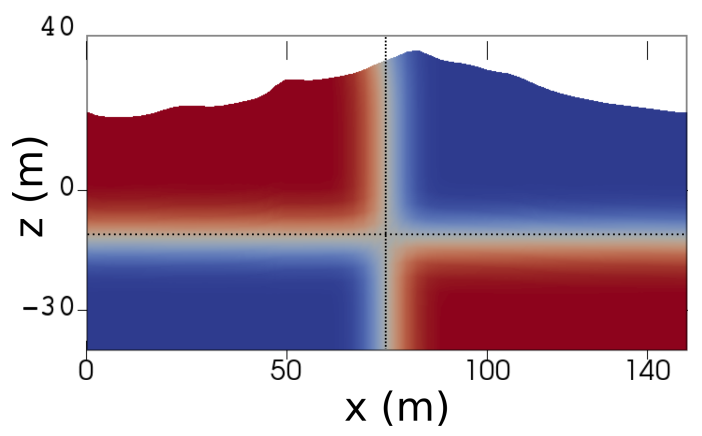

(c) True S-velocity Slice at $y=160 \mathrm{~m}$

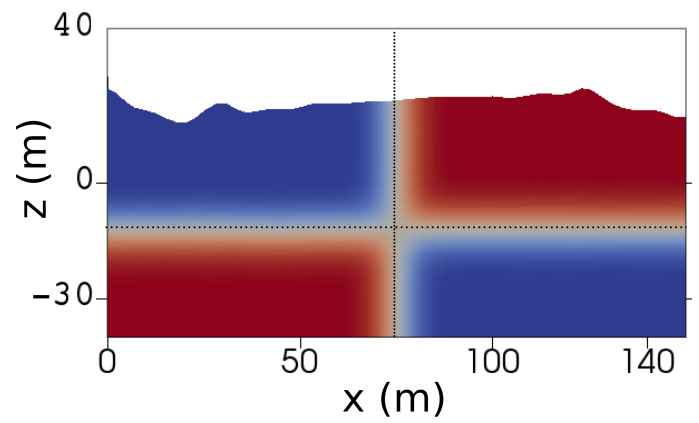

(b) Inverted S-velocity Slice at $y=80 \mathbf{m}$

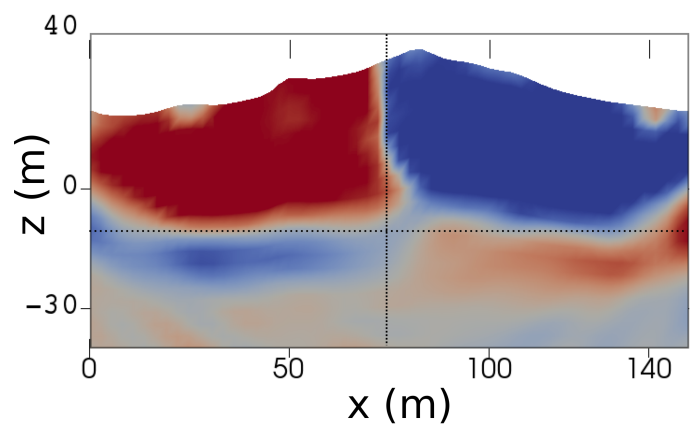

(d) Inverted S-velocity Slice at $y=160 \mathrm{~m}$

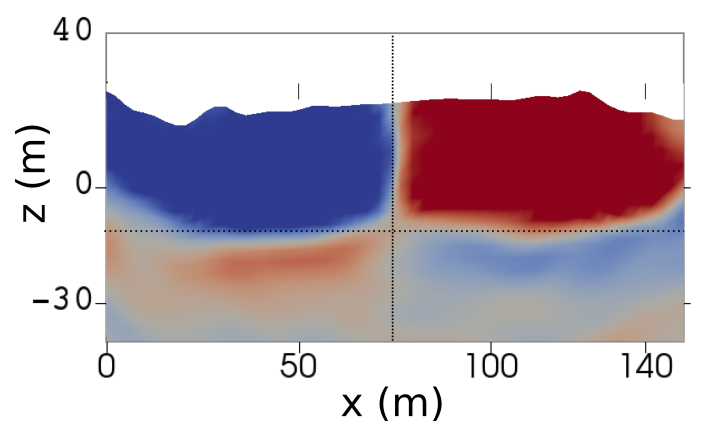

Figure 7: True S-velocity slices at $y=($ a) $80 \mathrm{~m}$ and (c) $160 \mathrm{~m}$. Inverted S-velocity slices at $y=(\mathrm{b}) 80 \mathrm{~m}$ and (d) $160 \mathrm{~m}$. 
(a) Dispersion Curves at Source A

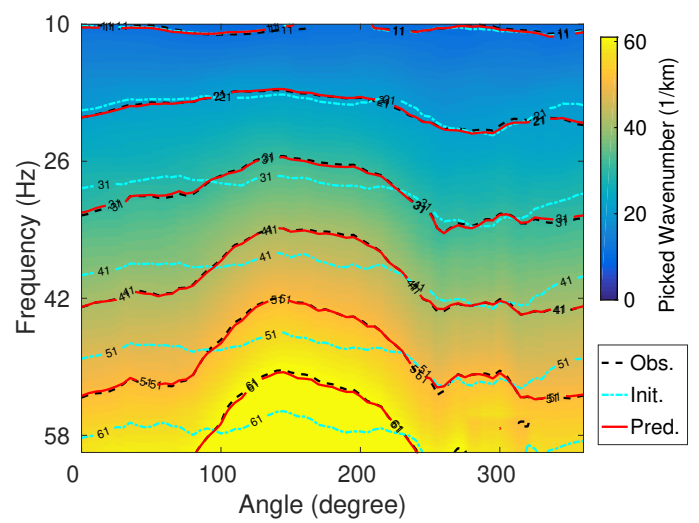

(b) Dispersion Curves at Source B

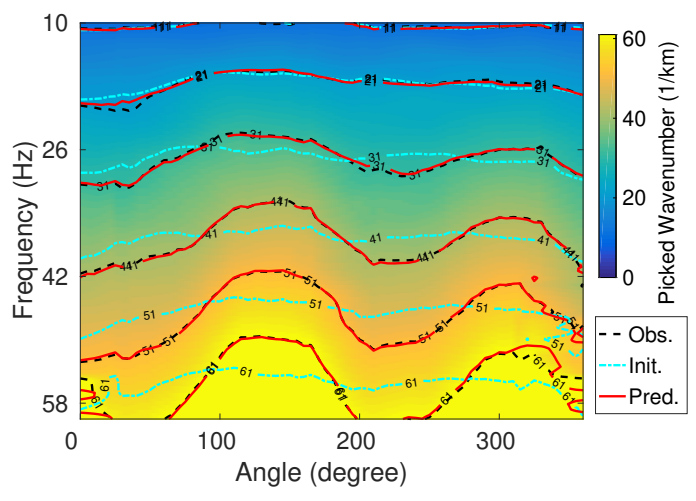

Figure 8: Observed dispersion curves from the CSGs with their sources located at points (a) A and (b) B (indicated in Figure 3a), where the black dashed lines, the cyan and red dash-dot lines represent the contours of the observed, initial and inverted dispersion curves, respectively. 


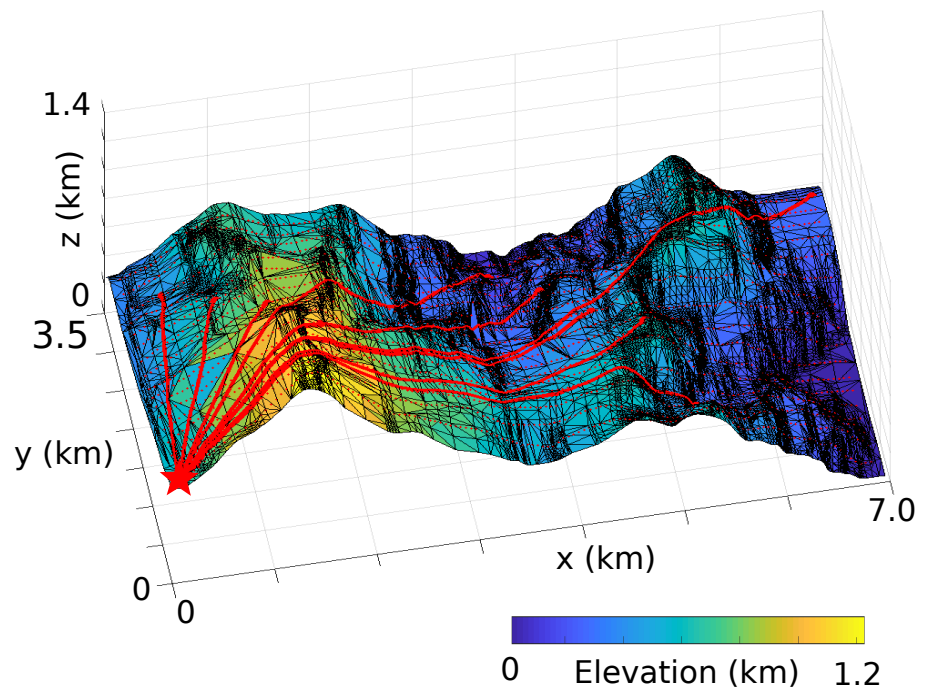

Figure 9: Topography of the 3D Foothill model, where the red lines are the geodesic paths for the source marked by the red star. 
(a) True S-velocity Model

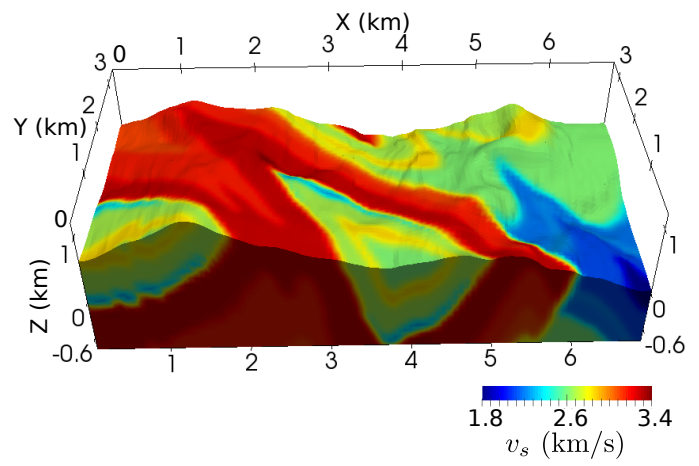

(c) Initial S-velocity Model

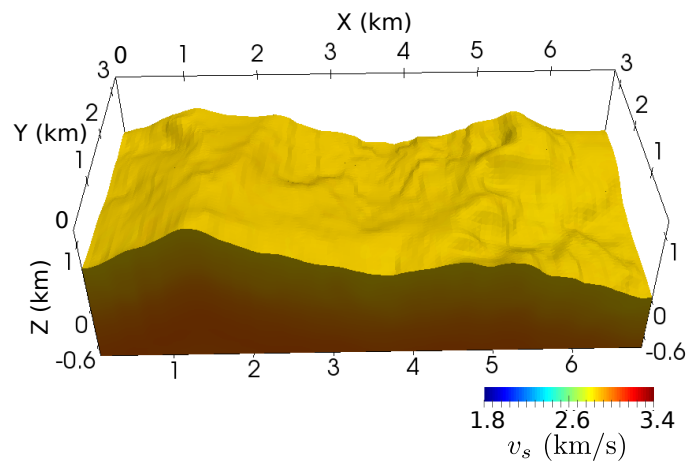

(b) Corresponding Mesh

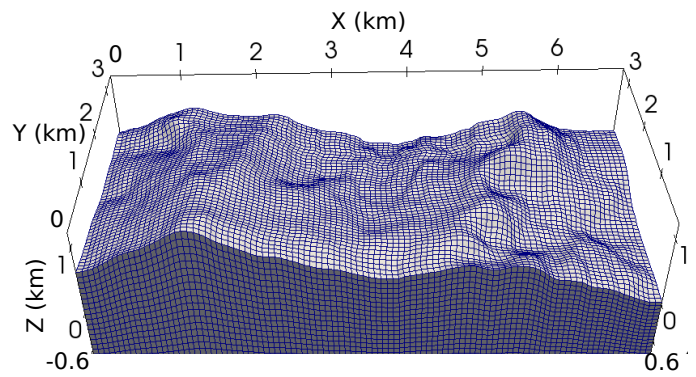

(d) S-velocity Tomogram

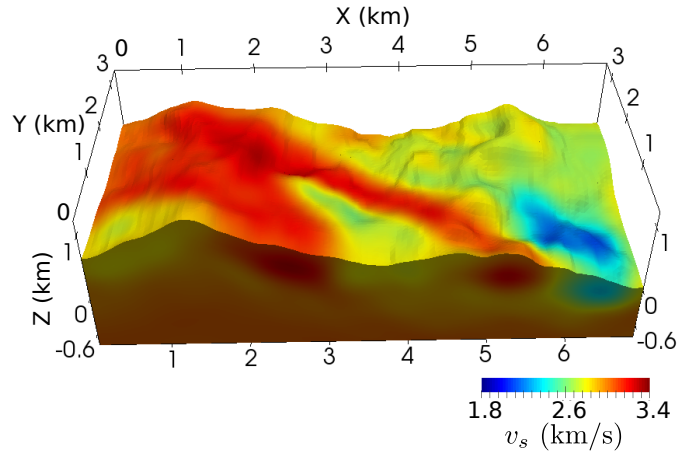

Figure 10: (a) True S-velocity model, (b) corresponding mesh, (c) initial S-velocity model and (d) S-velocity tomogram. 


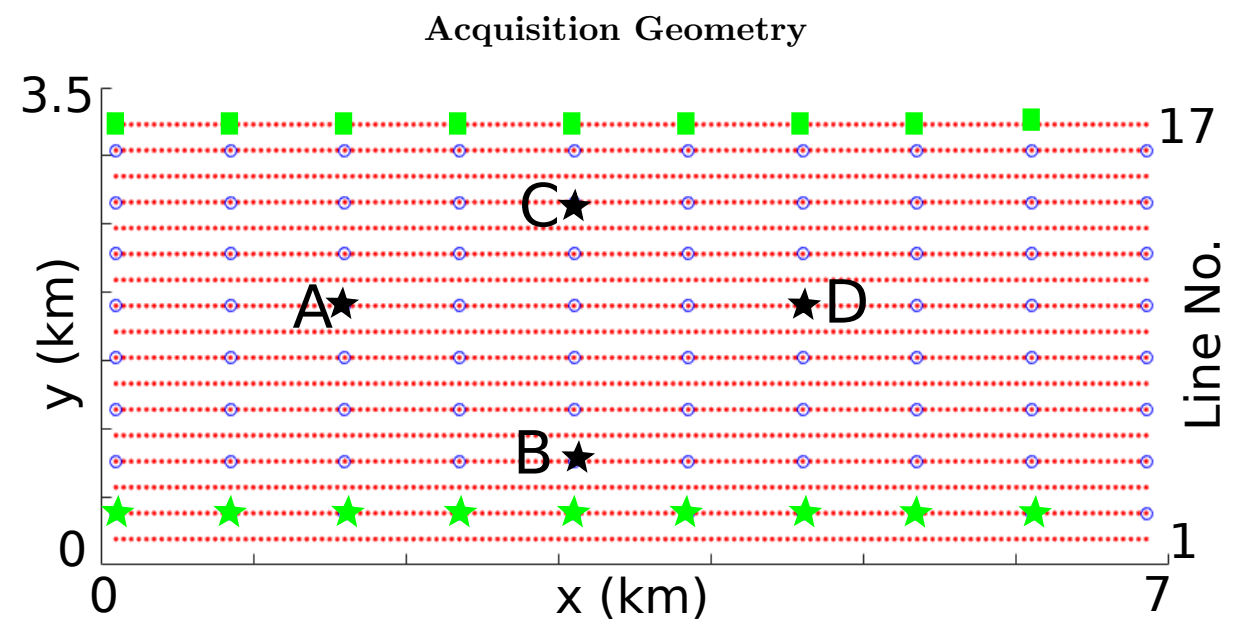

Figure 11: Acquisition geometry for the numerical tests with data generated for the 3D Foothill model, where the red dots and blue circles indicate the locations of the receivers and sources, respectively. 
(a) Dispersion Curves at Source A

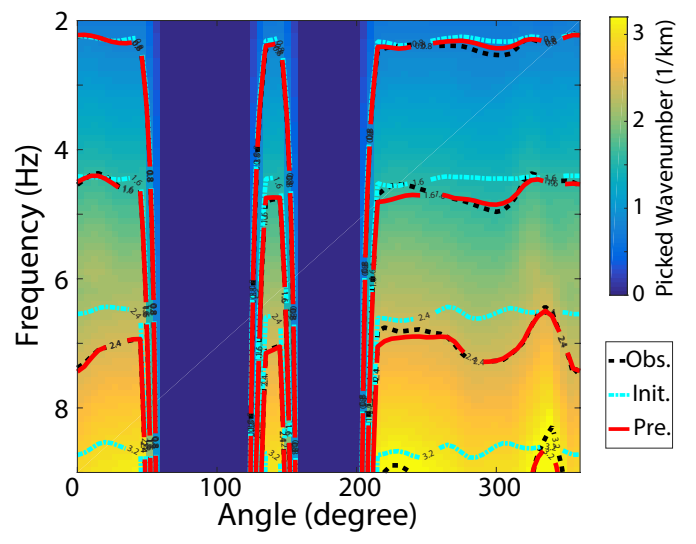

(c) Dispersion Curves at Source C

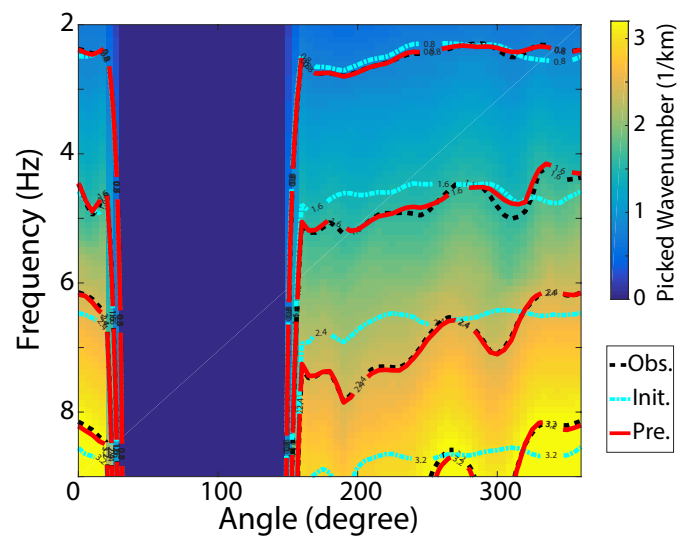

(b) Dispersion Curves at Source B

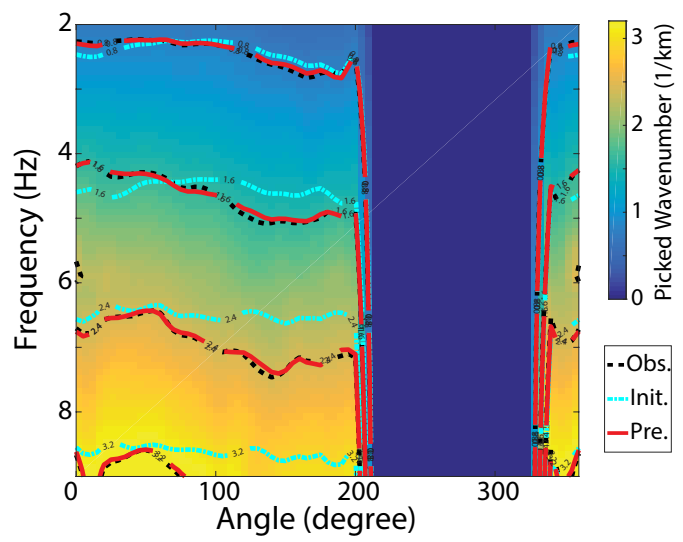

(d) Dispersion Curves at Source D

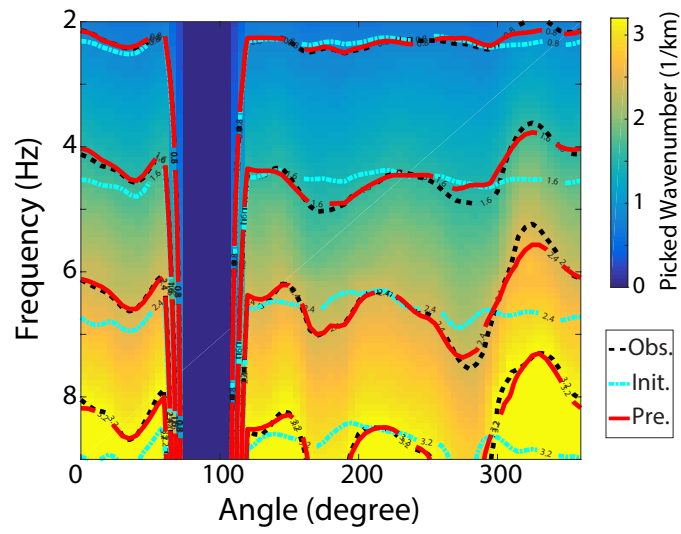

Figure 12: Observed dispersion curves for the sources located at (a) A, (b) B, (c) C and (d) D indicated in Figure 11b, where the black dashed lines, the cyan dash-dot lines and the red lines represent the contours of the observed, initial and inverted dispersion curves, respectively. 
(a) Slice of True Model at $y=433 \mathbf{m}$

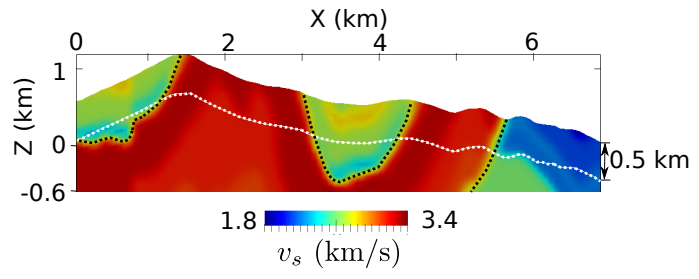

(b) Slice of Initial Model at $y=433 \mathrm{~m}$

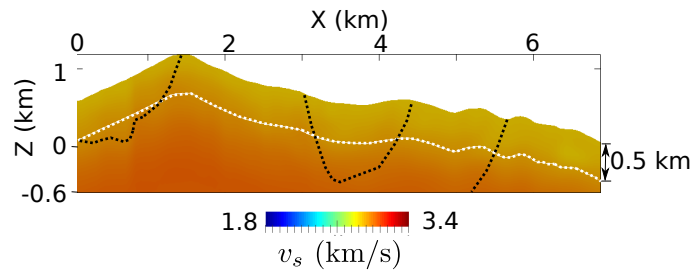

(c) Slice of Tomogram at $y=433 \mathrm{~m}$

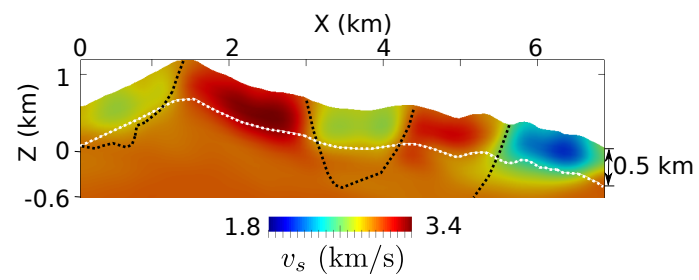

Figure 13: Slices of the (a) true, (b) initial, and (c) inverted S-velocity models at $y=433$ $\mathrm{m}$, where the black and white dashed lines indicate the large velocity contrast boundaries and the boundaries $0.5 \mathrm{~km}$ below the free surface, respectively. 
(a) Depth Slice of True Model

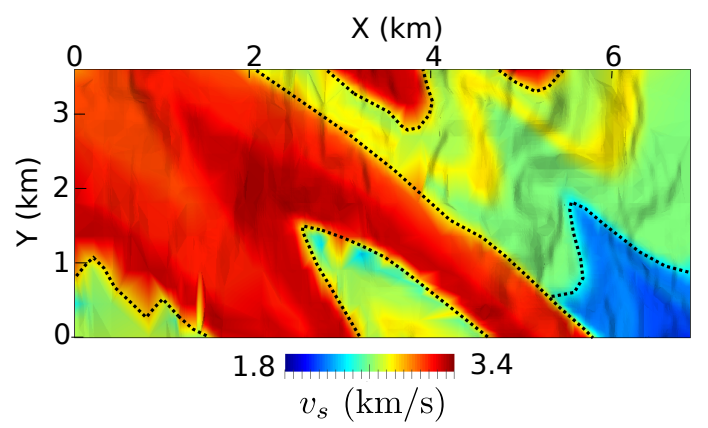

(b) Depth Slice of Initial Model

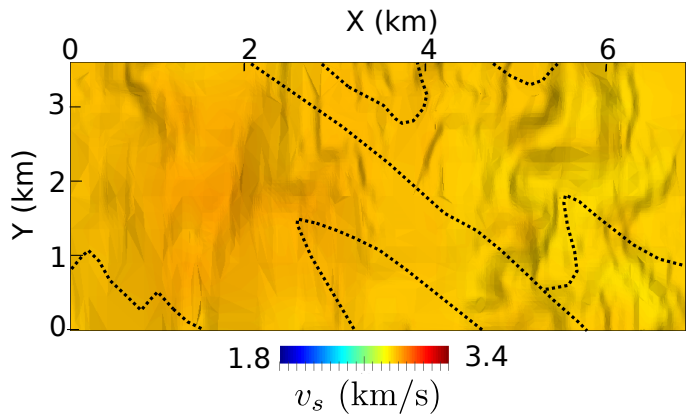

(c) Depth Slice of 3D Tomogram

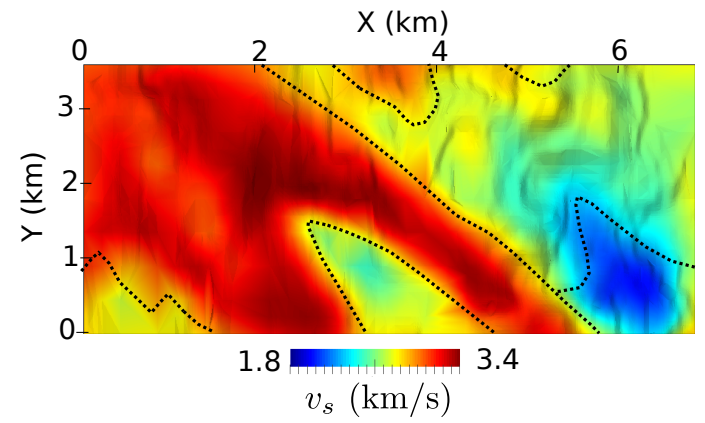

Figure 14: Depth slices $300 \mathrm{~m}$ below the surface for the (a) true, (b) initial and (c) inverted Foothill S-velocity models, where the black dashed lines indicate the large velocity contrast boundaries. 
(a) CSG B Line \# 17 from Initial Model

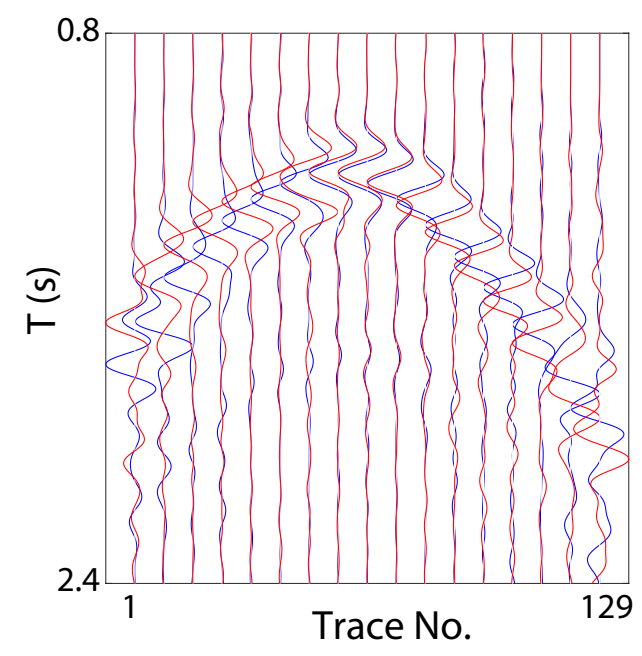

(c) CSG C Line \# 2 from Initial Model

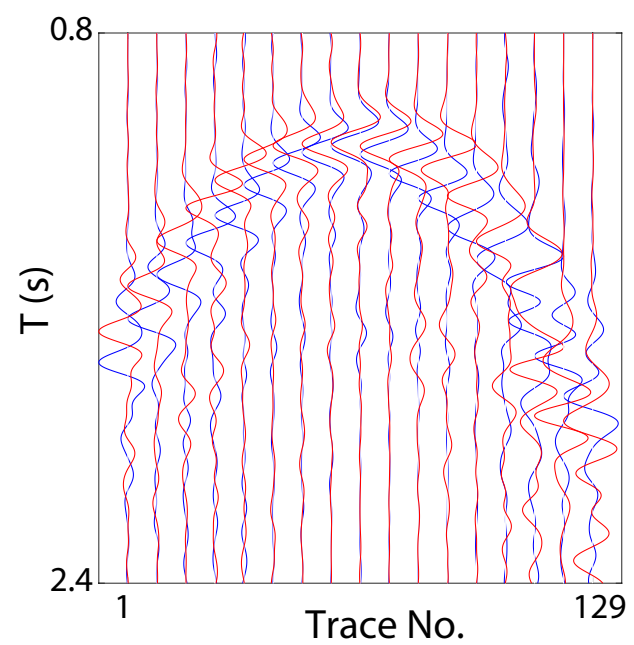

(b) CSG B Line \# 17 from 3D Tomogram

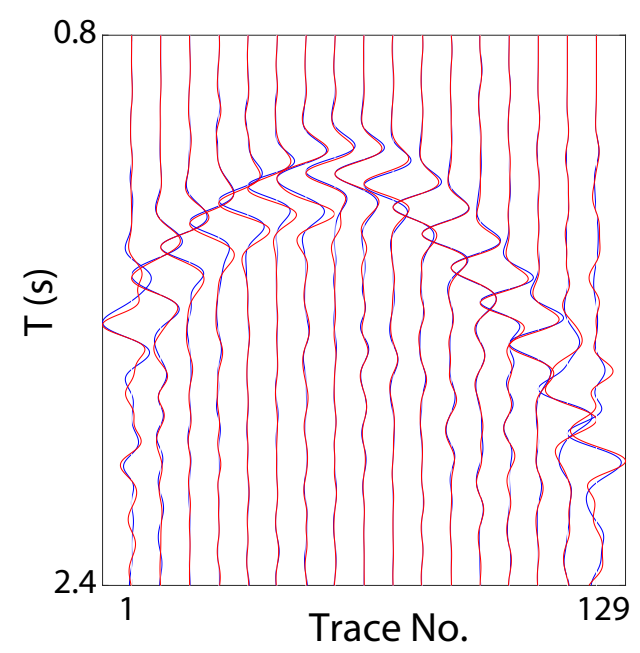

(d) CSG C Line \# 2 from 3D Tomogram

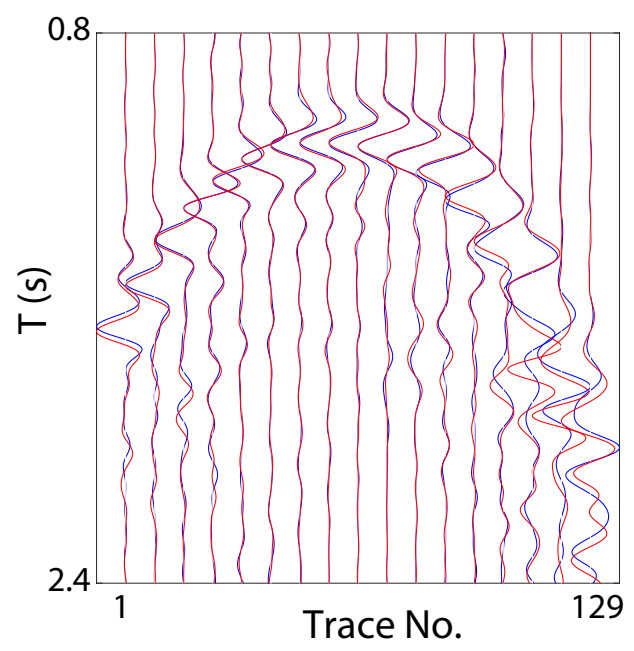

Figure 15: Comparison between the observed (red) and synthetic (blue) traces at far offsets predicted from the initial model (LHS panels) and 3D tomogram (RHS panels) for CSG B in (a) and (b), and CSG C in (c) and (d). Here, the locations of points B and C and the line numbers are indicated in Figure 11. 
(a) COG from Initial Model

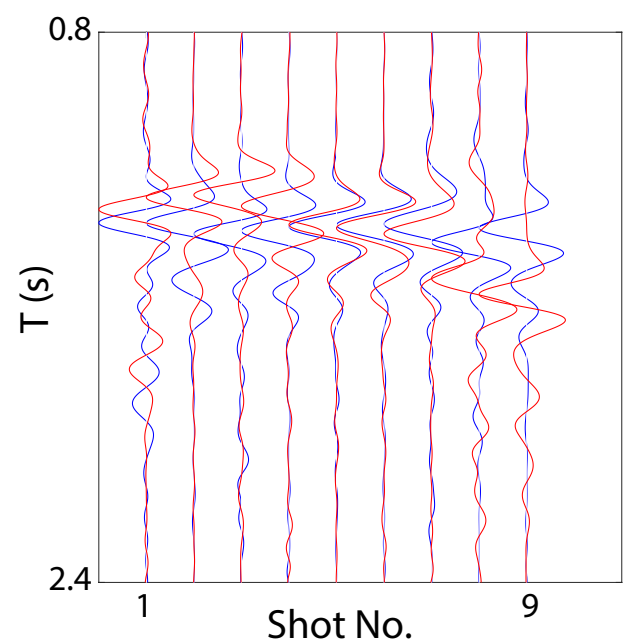

(b) COG from 3D Tomogram

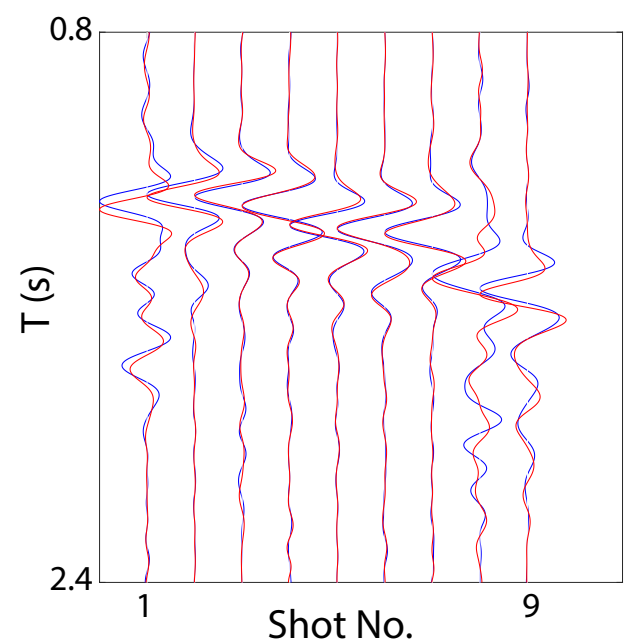

Figure 16: COGs with the offset of $2.85 \mathrm{~km}$, which are retrieved from the traces located at the green rectangles in Figure 11 of the CSGs with the sources located at the green stars in Figure 11. Here the red and blue wiggles represent the observed and predicted COGs, respectively. 
a) Geologic Map

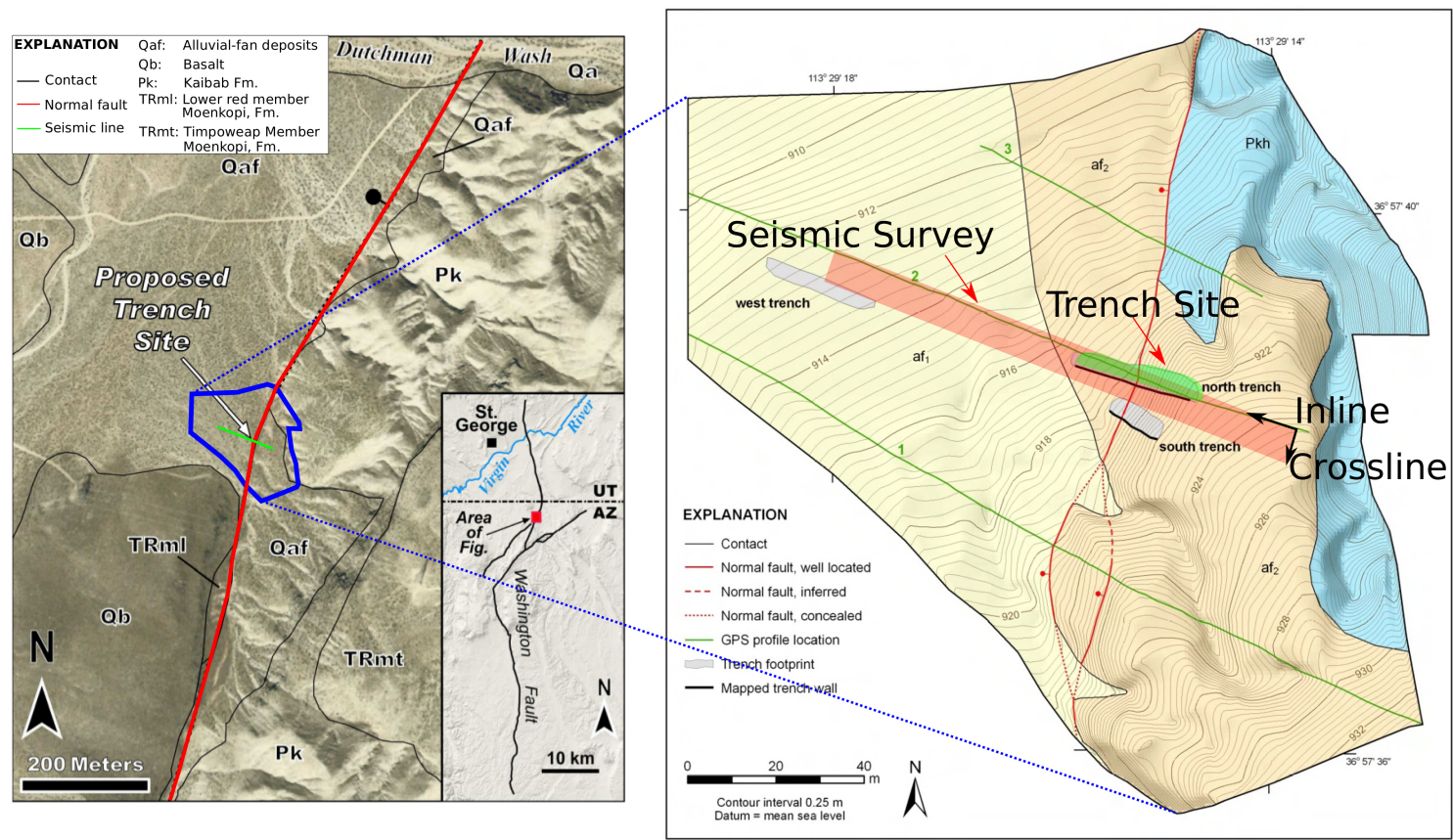

Figure 17: (a) Map of the Washington fault and the survey site. The location of the survey site is $5 \mathrm{~km}$ south of the Utah-Arizona border. (b) Topographic map around the seismic survey, where the red and green rectangles indicate the locations of the 3D seismic survey and the trench site, respectively. 


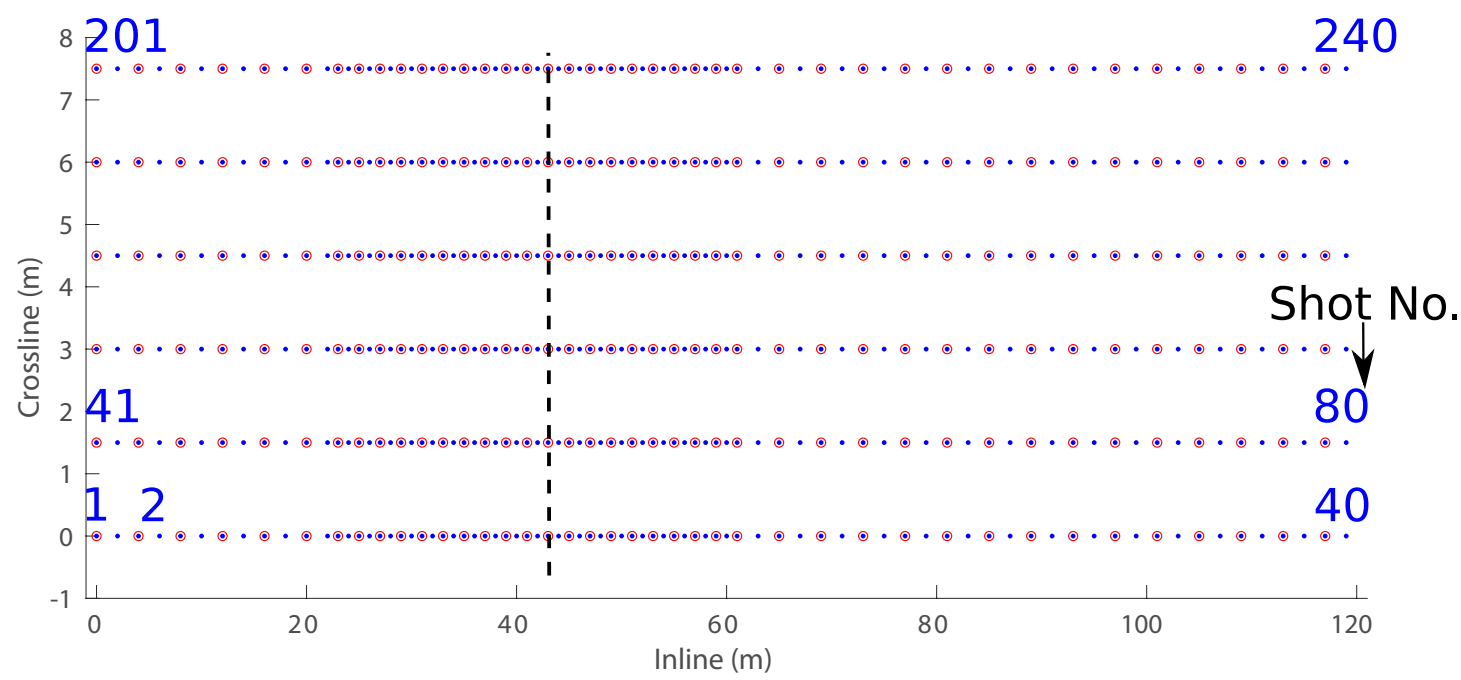

Figure 18: Survey geometry for the 3D experiment in the Washington fault zone. The open red circles denote the locations of sources and the solid blue dots denote the locations of receivers. The dashed black line denotes the location of the fault scarp. 


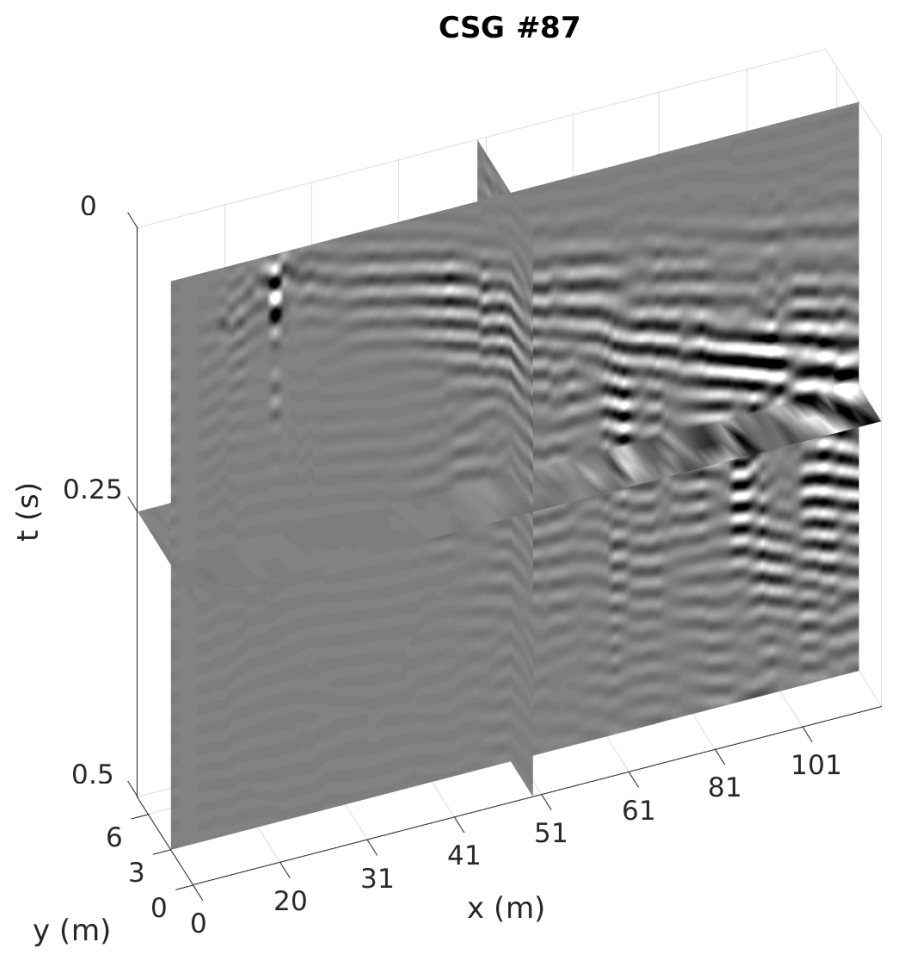

Figure 19: Common shot gather \# 87 of Washington fault data. 


\section{a) Traveltime before Correction b) Traveltime after Correction}
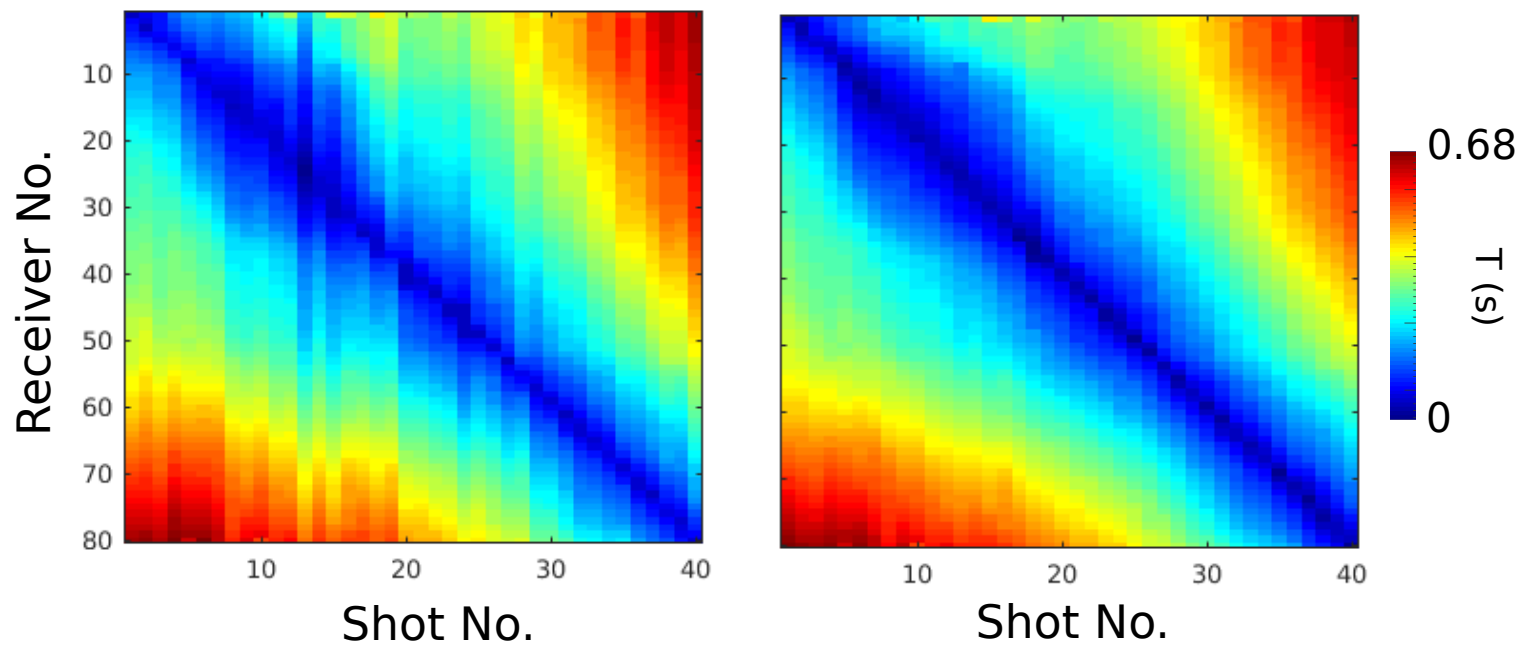

Figure 20: Traveltime matrices before and after the correction of the acquisition hardware error for the $2 \mathrm{D}$ data set on line \#4. 
(a) Dispersion Curves for $\theta=0^{\circ}$

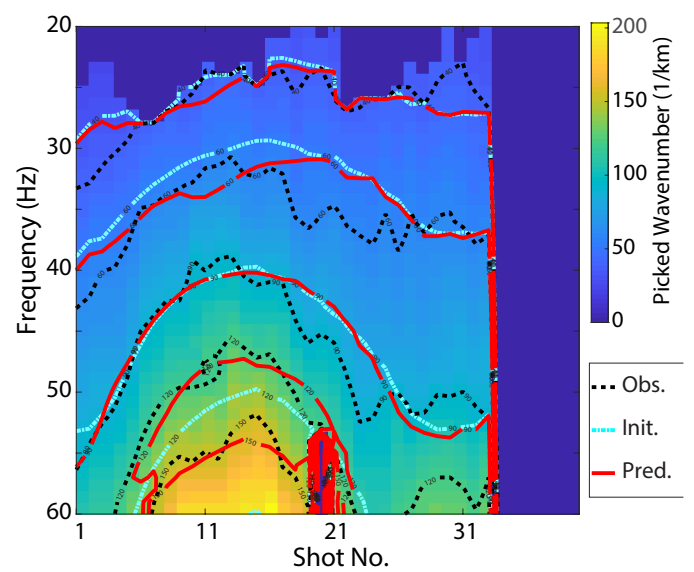

(b) Dispersion Curves for $\theta=180^{\circ}$

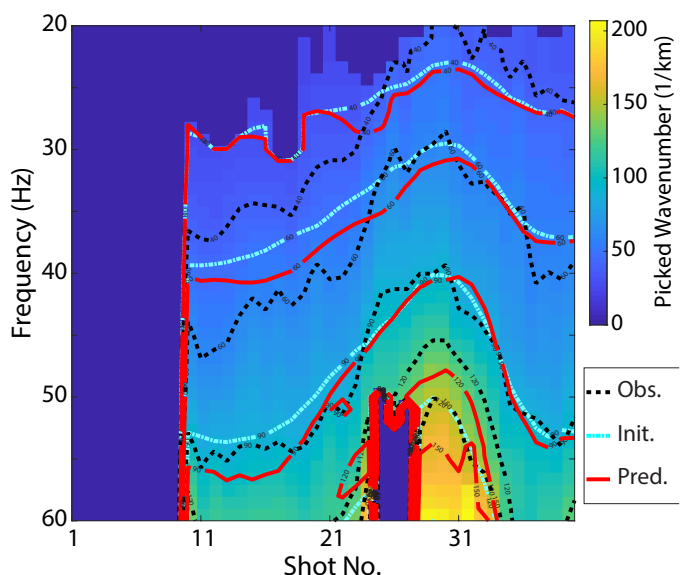

Figure 21: (a) Observed dispersion curves for the CSGs on Line \# 4 along the azimuthal angles (a) $\theta=0^{\circ}$ and (b) $\theta=180^{\circ}$, where the black dashed lines, the cyan dash-dot lines and the red lines represent the contours of the observed, initial and inverted dispersion curves, respectively. 

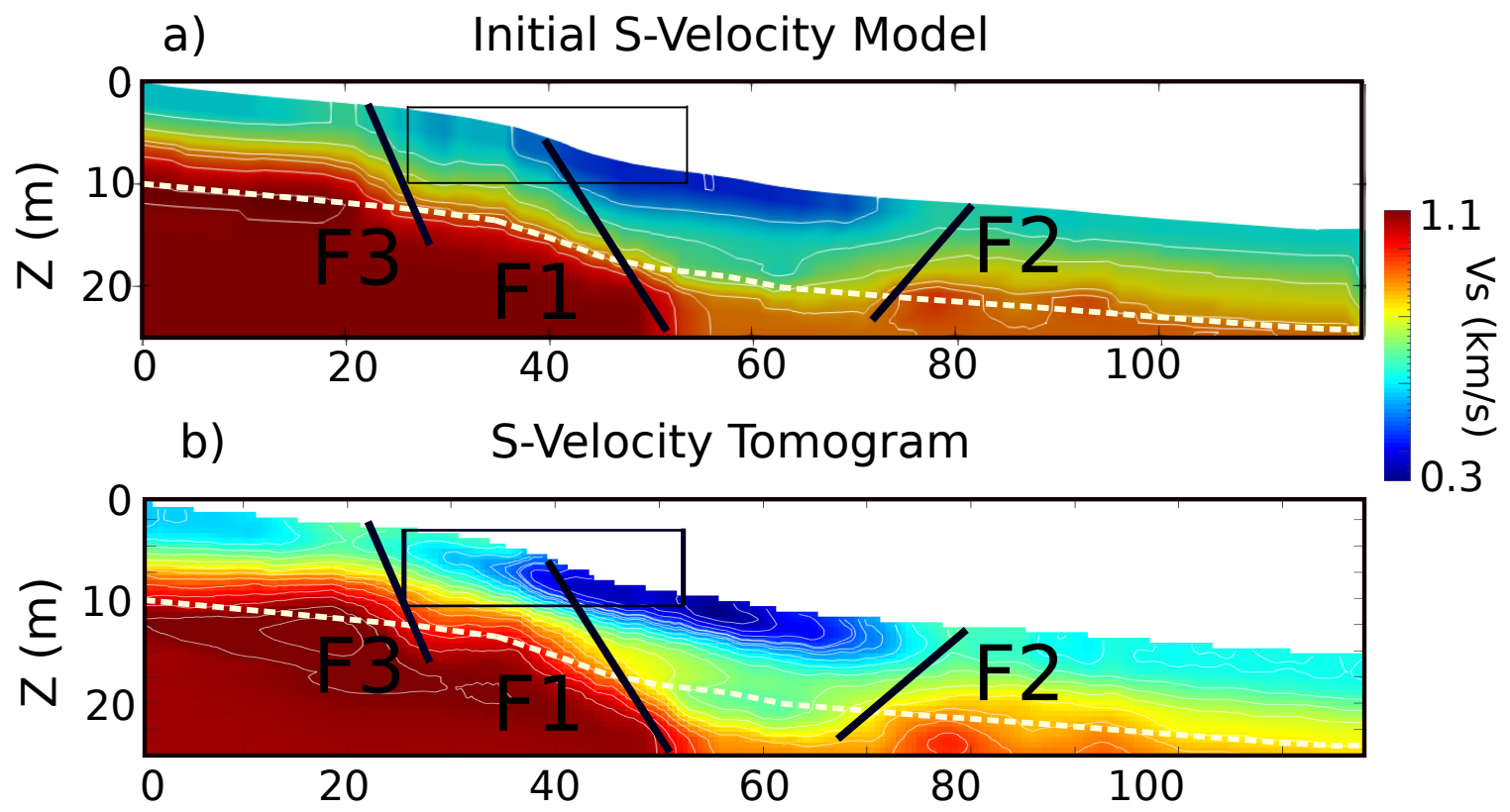

c) P-Velocity Tomogram

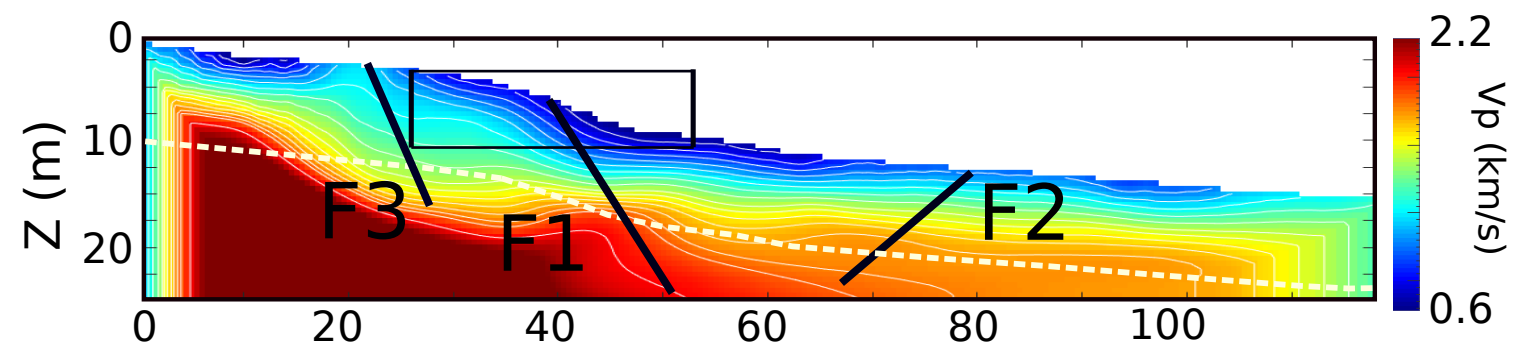

d) $\quad$ Vp/Vs Ratio Map

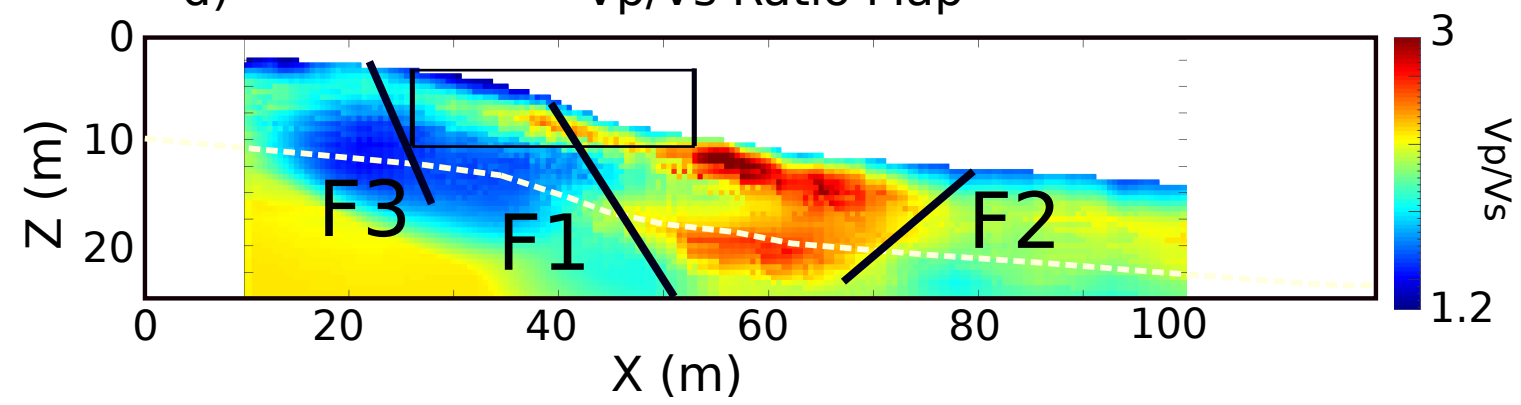

Figure 22: (a) Initial and (b) inverted S-wave velocity models beneath line \#4. (c) P-wave velocity tomogram calculated from the picked traveltimes in Figure 20b. (d) Vp/Vs ratio tomogram beneath line \#4. Here the white lines indicate the boundaries $10 \mathrm{~m}$ below the free surface. The trench is excavated in the locations of the black rectangles. The lines labeled with "F1" and "F2" are interpreted as the locations of the main fault and the antithetic fault. The line labeled with "F3" is the location of another possible fault. "CW" represents the colluvial wedge. 
(a) Initial S-velocity Model

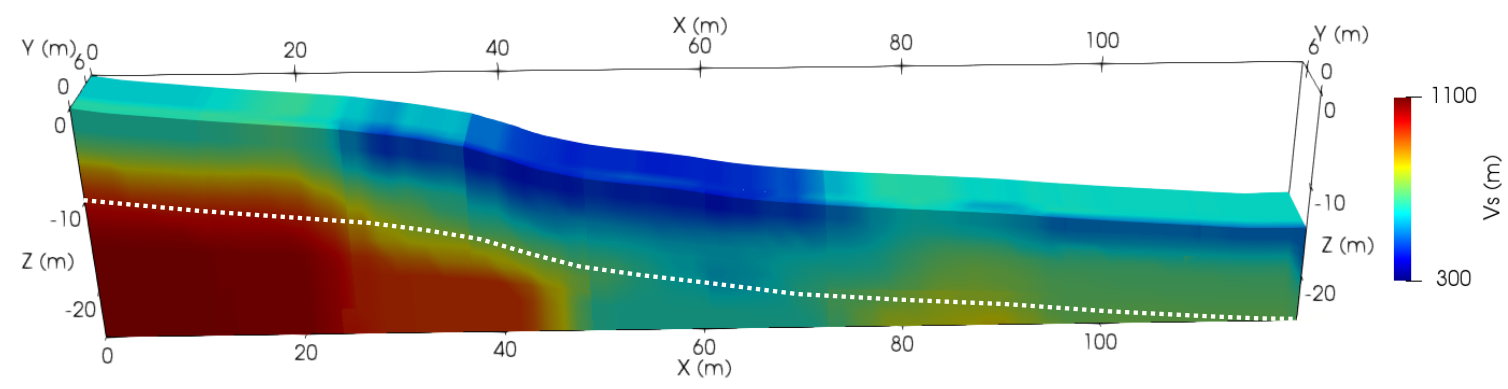

(b) 2D S-velocity Tomogram

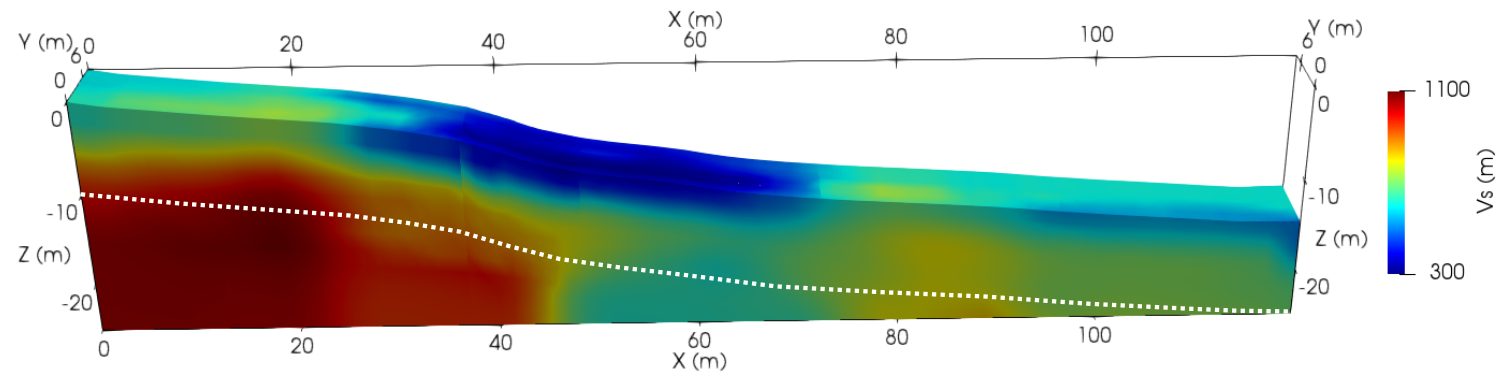

(c) 3D S-velocity Tomogram

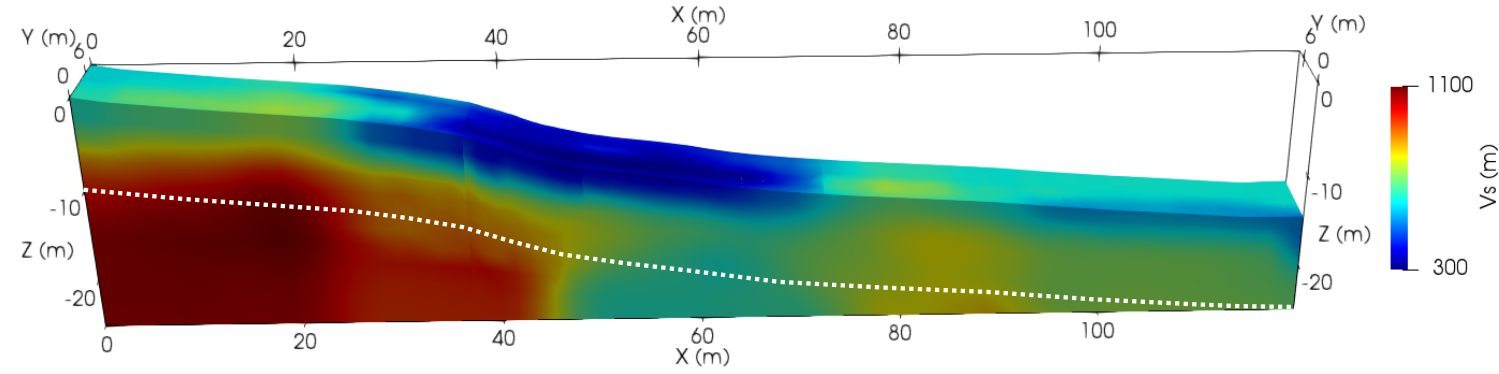

Figure 23: (a) Initial, (b) 2D and (c) 3D S-wave velocity tomograms. Here, the depth and S-wave velocity of the initial model are calculated by scaling the wavelength and phase velocity with factors of 0.5 and 1.1, respectively (Liu et al., 2019). 3D TWD cannot make any further update for the 2D TWD tomogram so that the 3D TWD tomogram in (a) is almost the same as the 2D TWD tomogram in (b). 
(a) CSG from Initial S-velocity Model

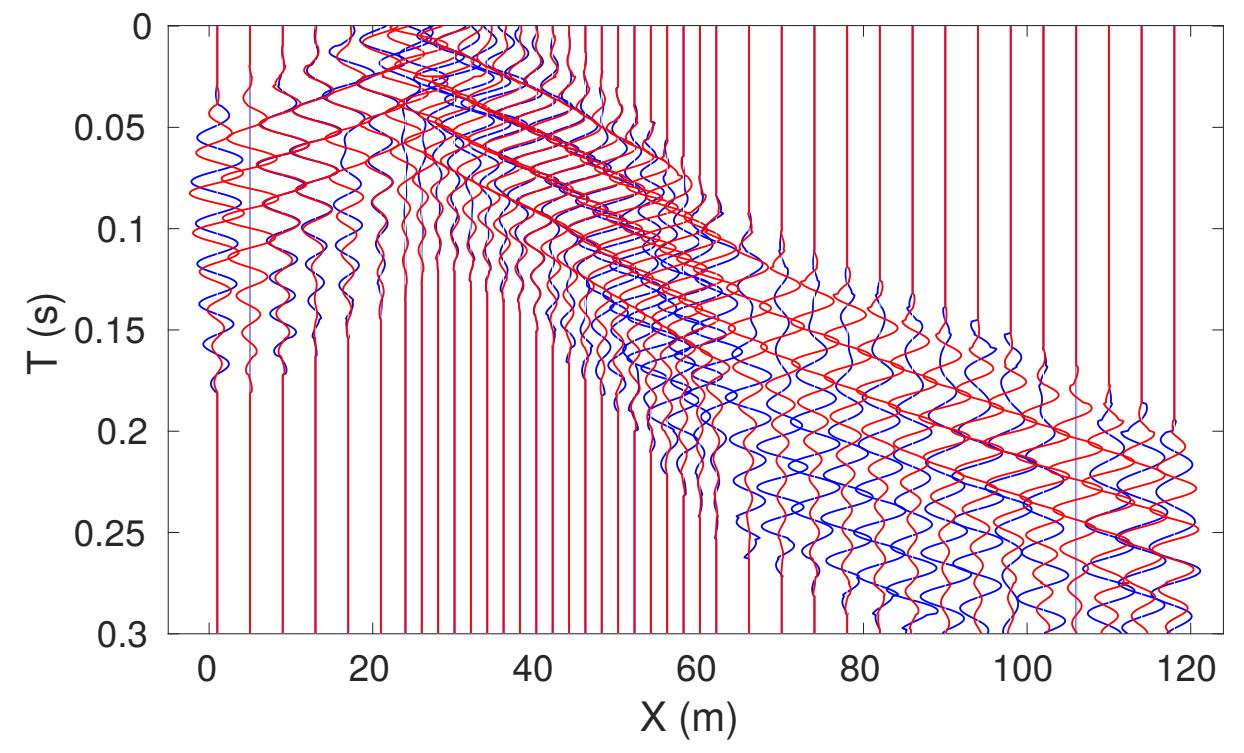

(b) CSG from the Inverted S-velocity Model

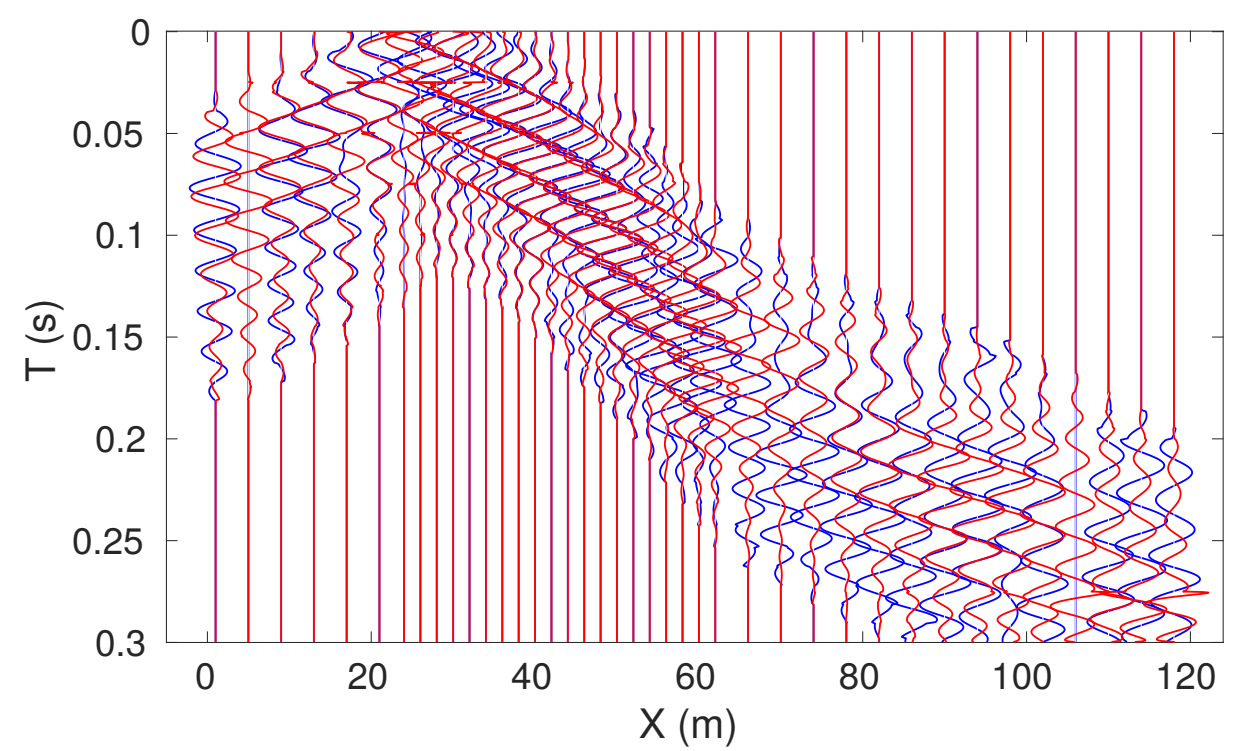

Figure 24: Comparison between the observed (blue) and synthetic (red) traces predicted from the (a) initial and (b) inverted S-velocity models for CSG \# 128. 
(a) COG from Initial Model

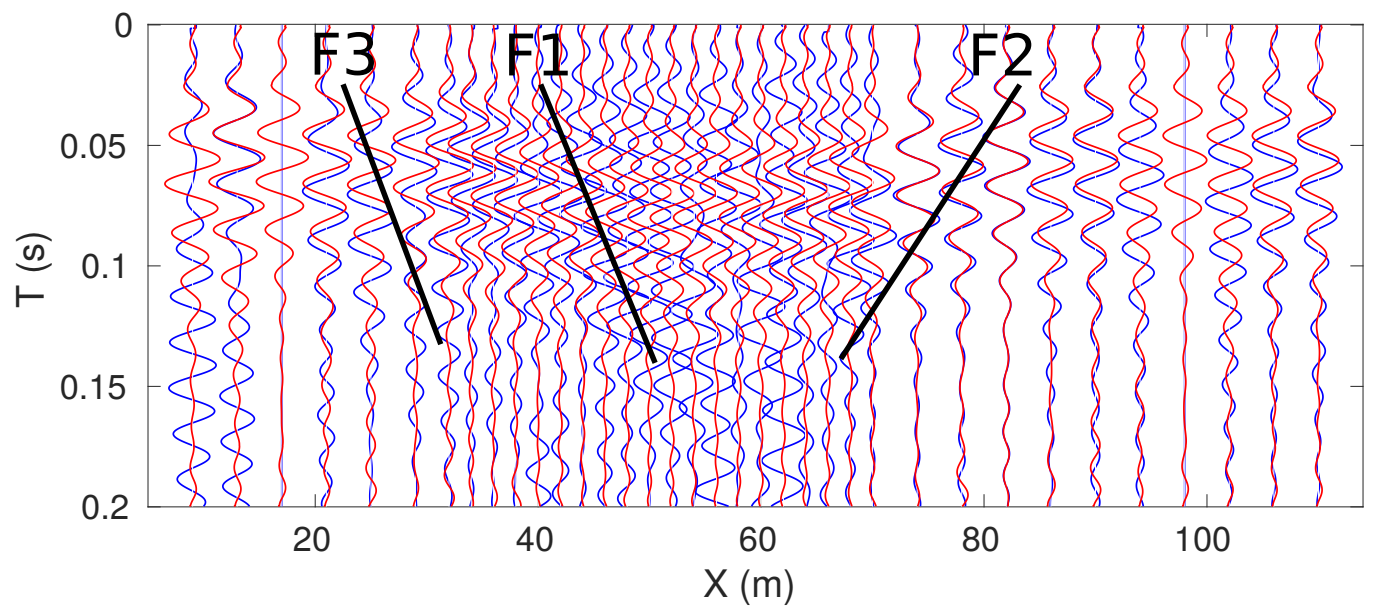

(b) COG from Inverted Model

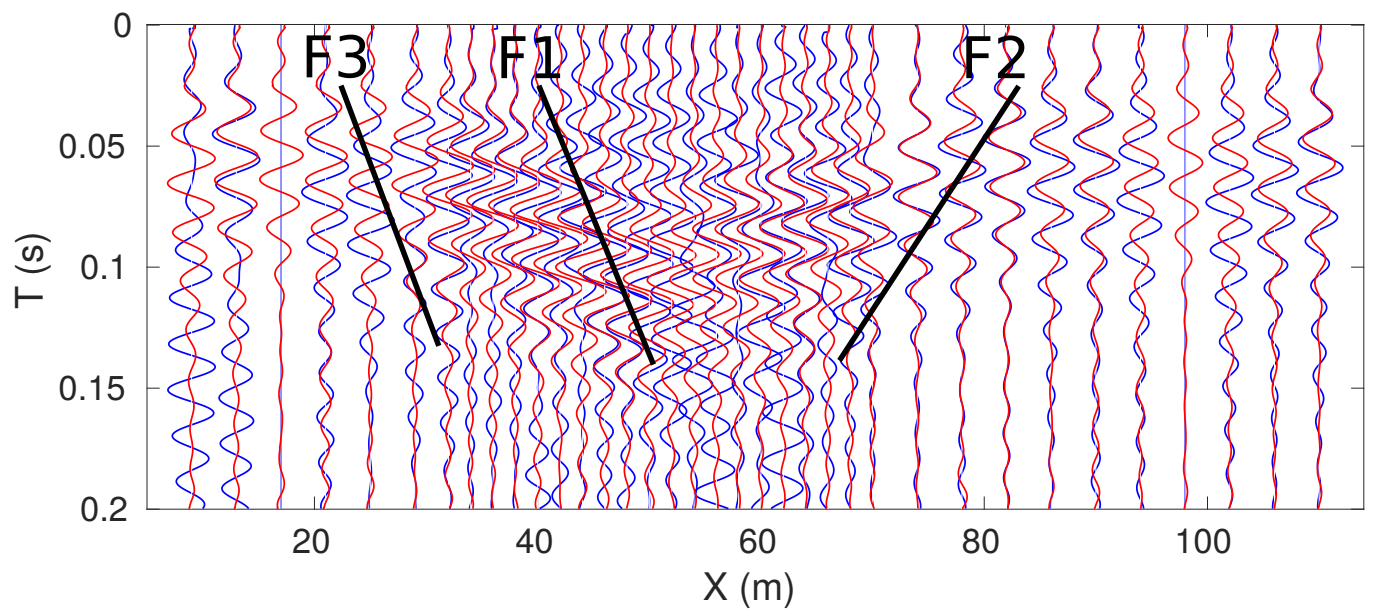

Figure 25: COGs with the offset of $16 \mathrm{~m}$ for line \# 4 calculated from the (a) initial and (b) inverted S-velocity models, where the blue and red wiggles represent the observed and predicted COGs, respectively. 
S-velocity Tomogram with COG

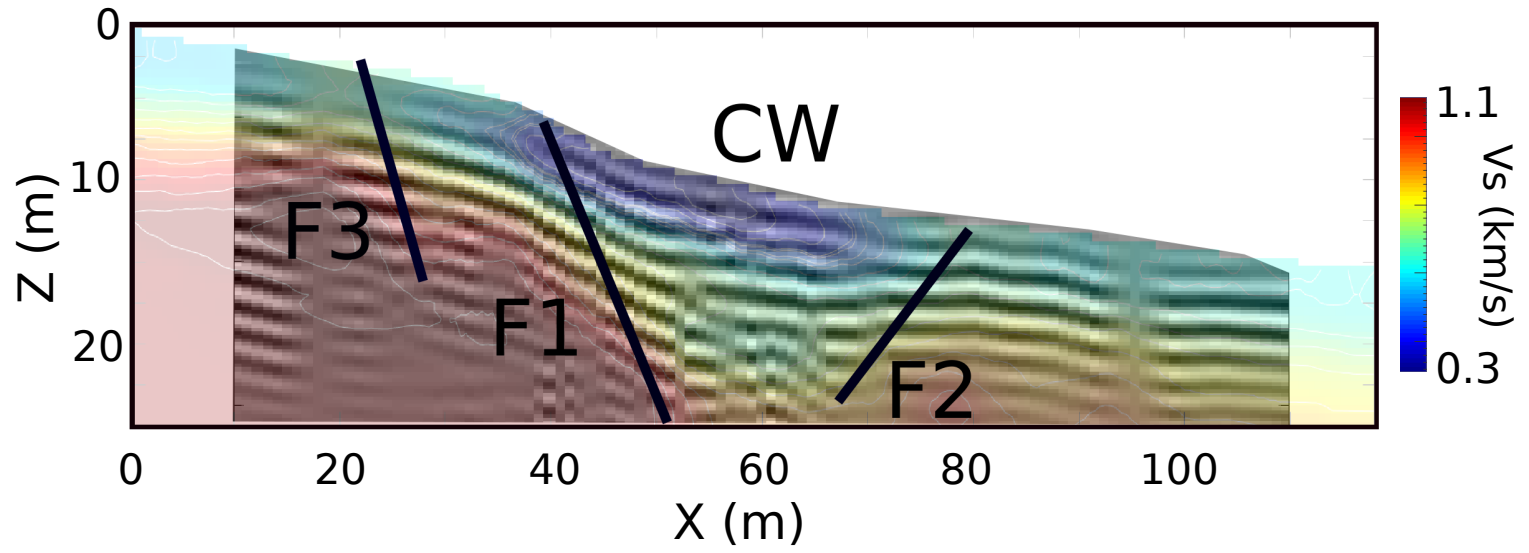

Figure 26: Observed COGs with the offset of $16 \mathrm{~m}$ are superposed on the S-velocity tomogram, where the COGs are adjusted by following the topography. 
a) Zoom View of S-velocity Tomogram

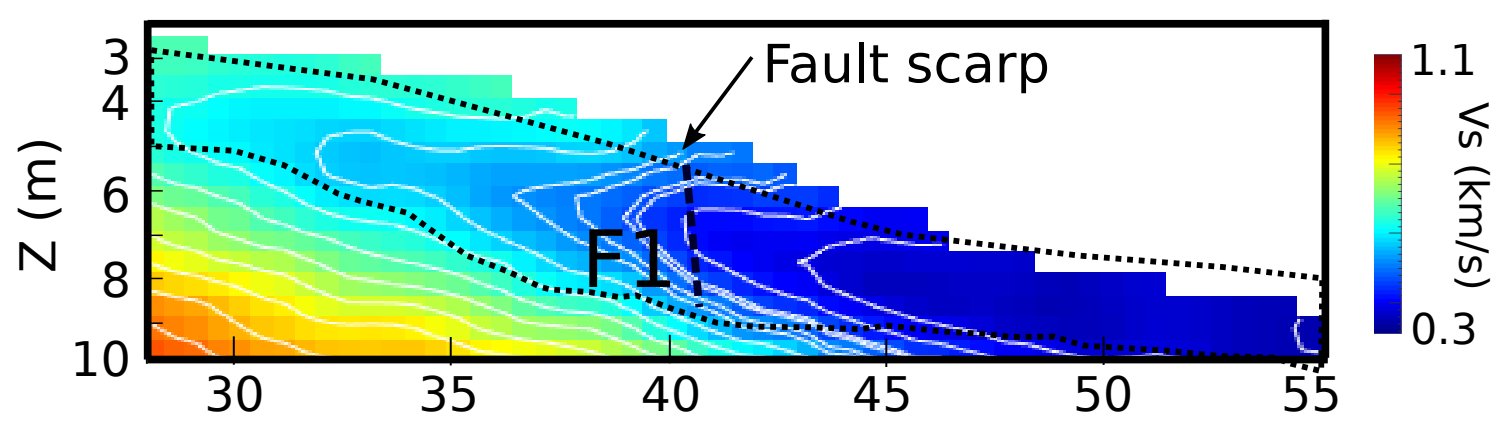

b) Zoom View of P-velocity Tomogram

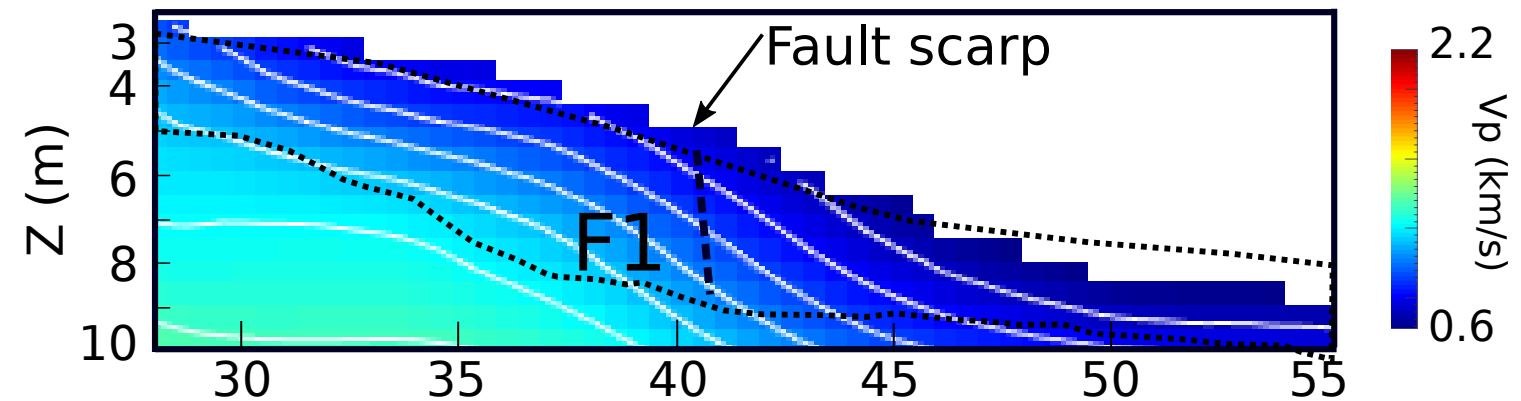

c) Zoom View of Vp/Vs Tomogram

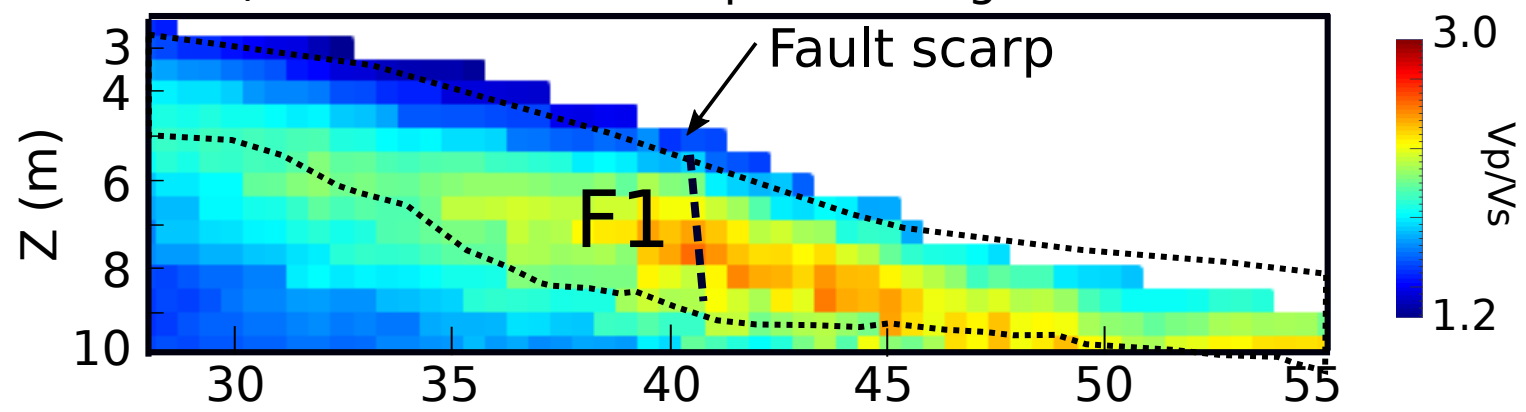

d) Trench Log

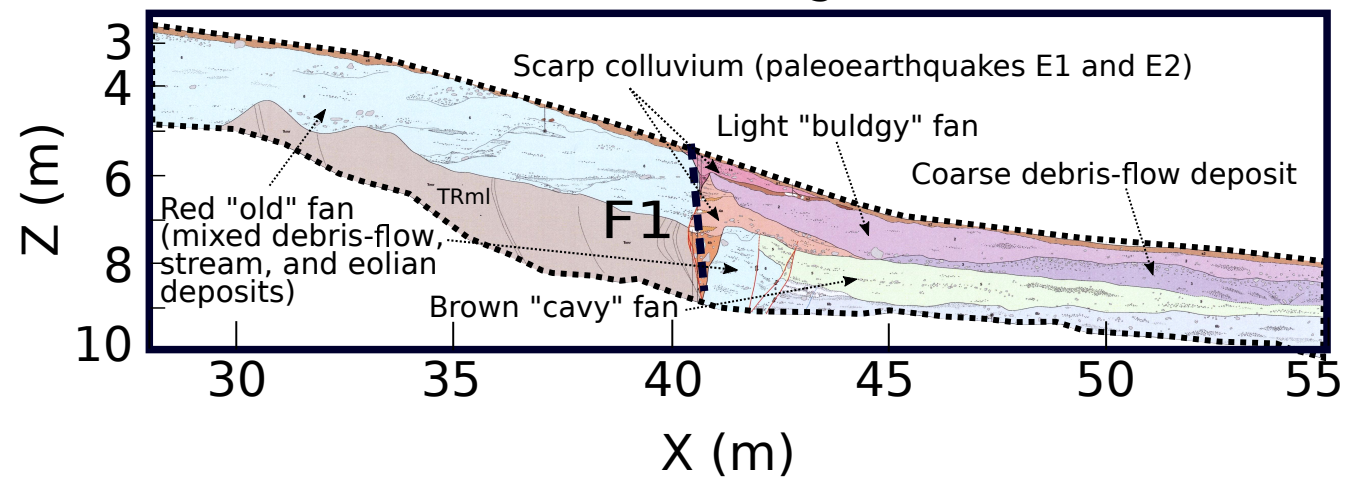

Figure 27: Zoom views of (a) S-velocity and (b) P-velocity tomograms and (c) Vp/Vs tomogram in Figure 22. (d) Ground truth extracted from a nearby trench log (Hanafy et al., 2015). 
(a) A geodesic on a simple surface mesh

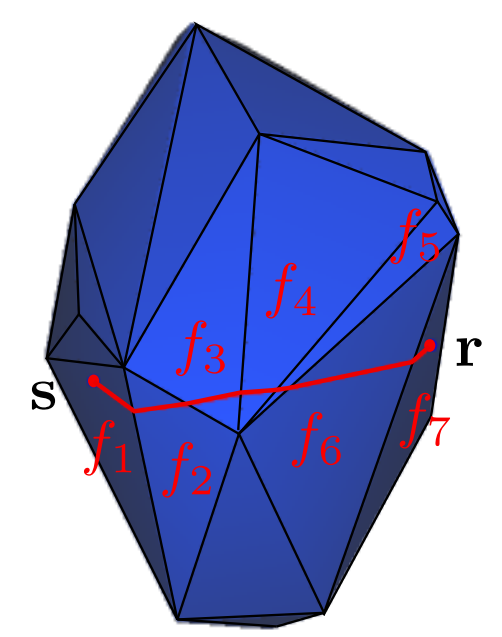

(b) The same geodesic, with its faces unfolded into the plane.

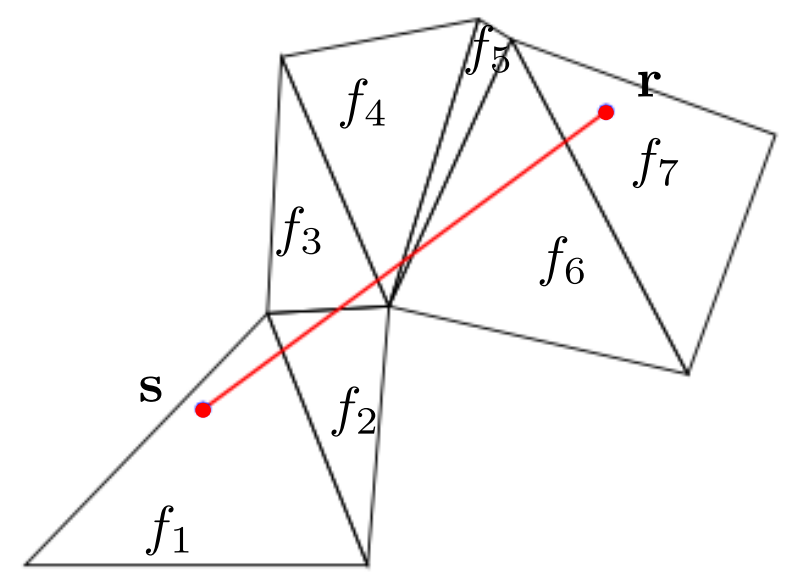

Figure 28: Schematic diagram of the calculation of the geodesic on a simple surface mesh by unfolding. 